\title{
Article \\ On Fuzzy Extended Hexagonal $b$-Metric Spaces with Applications to Nonlinear Fractional Differential Equations
}

\author{
Sumaiya Tasneem Zubair ${ }^{1}\left(\mathbb{D}\right.$, Kalpana Gopalan ${ }^{1, *(\mathbb{D})}$, Thabet Abdeljawad ${ }^{2,3} * \mathbb{D}$ and Bahaaeldin Abdalla ${ }^{2} \mathbb{D}$ \\ 1 Department of Mathematics, Sri Sivasubramaniya Nadar College of Engineering, Kalavakkam, \\ Chennai 603 110, India; sumaiyatasneemz@ssn.edu.in \\ 2 Department of Mathematics and Sciences, Prince Sultan University, P.O. Box 66833, \\ Riyadh 11586, Saudi Arabia; babdallah@psu.edu.sa \\ 3 Department of Medical Research, China Medical University, Taichung 40402, Taiwan \\ * Correspondence: kalpanag@ssn.edu.in (K.G.); tabdeljawad@psu.edu.sa (T.A.)
}

Citation: Zubair, S.T.; Gopalan, K.; Abdeljawad, T.; Abdalla, B. On Fuzzy Extended Hexagonal $b$-Metric Spaces with Applications to Nonlinear Fractional Differential Equations. Symmetry 2021, 13, 2032. https:// doi.org/10.3390/sym13112032

Academic Editor: Alexander Zaslavski

Received: 23 September 2021 Accepted: 20 October 2021 Published: 27 October 2021

Publisher's Note: MDPI stays neutral with regard to jurisdictional claims in published maps and institutional affiliations.

Copyright: (c) 2021 by the authors. Licensee MDPI, Basel, Switzerland. This article is an open access article distributed under the terms and conditions of the Creative Commons Attribution (CC BY) license (https:// creativecommons.org/licenses/by/ $4.0 /)$.

\begin{abstract}
The focus of this research article is to investigate the notion of fuzzy extended hexagonal $b$-metric spaces as a technique of broadening the fuzzy rectangular $b$-metric spaces and extended fuzzy rectangular $b$-metric spaces as well as to derive the Banach fixed point theorem and several novel fixed point theorems with certain contraction mappings. The analog of hexagonal inequality in fuzzy extended hexagonal $b$-metric spaces is specified as follows utilizing the function $b(c, d)$ : $\mathfrak{m}_{\mathrm{h}}(c, d, t+s+u+v+w) \geq \mathfrak{m}_{\mathrm{h}}\left(c, e, \frac{t}{b(c, d)}\right) * \mathfrak{m}_{\mathrm{h}}\left(e, f, \frac{s}{b(c, d)}\right) * \mathfrak{m}_{\mathrm{h}}\left(f, g, \frac{u}{b(c, d)}\right) * \mathfrak{m}_{\mathrm{h}}\left(g, k, \frac{v}{b(c, d)}\right) *$ $\mathfrak{m}_{\mathrm{h}}\left(k, d, \frac{w}{b(c, d)}\right)$ for all $t, s, u, v, w>0$ and $c \neq e, e \neq f, f \neq g, g \neq k, k \neq d$. Further to that, this research attempts to provide a feasible solution for the Caputo type nonlinear fractional differential equations through effective applications of our results obtained.
\end{abstract}

Keywords: fuzzy extended hexagonal $b$-metric spaces; fixed points; nonlinear fractional differential equation of the Caputo type

\section{Introduction and Preliminaries}

Following Banach's significant approach to fixed point theory based on the fixed point concept, the majority of the authors offered several research studies on this topic. Zadeh [1] proposed a fuzzy set in 1965, which generalised the notion of the crisp set by combining all elements with membership values in the interval [0,1]. Following the implementation of Zadeh's fuzzy topic, many researchers [2-5] expanded on the fuzzy metric area of study and developed certain results. In 1975, Kramosil and Michalek [6] brought the theory of fuzzy metric spaces. Grabiec [7] established the fuzzy form of Banach contraction mapping principle.

On the other hand, Bakhtin [8], Bourbaki [9], and Czerwik [10] all contributed to the development of the ideology of fixed points for $b$-metric spaces. Kamran et al. [11] implemented the conception of extended $b$-metric spaces, whereas, in [12], Mehmood et al. adapted fuzzy set theory to the definition of Kamran's by proposing the notion of an extended fuzzy $b$-metric spaces and proving various fixed point theorems on this space. In an analogous way, the researchers in [13-16] identified the fuzzy form of rectangular b-metric spaces [17], controlled metric type spaces [18], double controlled metric type spaces [19], and triple controlled metric type spaces [20]. Very recently, the idea of extended hexagonal $b$-metric spaces was initiated by Kalpana et al. [21] and several fixed point theorems under different contraction mappings were obtained on this metric. For more results on fuzzy metric spaces, the readers may refer to [22-27].

Inspired by all of the aforementioned facts, the significant aim of this research is to find an appropriate fuzzy metric space using a control function $b: X \times X \rightarrow[1, \infty)$ resulting in the new fuzzy inequality form: 


$$
\begin{aligned}
\mathfrak{m}_{\mathrm{h}}(c, d, b(c, d)(t+s+u+v+w)) \geq & \mathfrak{m}_{\mathrm{h}}(c, e, t) * \mathfrak{m}_{\mathrm{h}}(e, f, s) * \mathfrak{m}_{\mathrm{h}}(f, g, u) * \mathfrak{m}_{\mathrm{h}}(g, k, v) \\
& * \mathfrak{m}_{\mathrm{h}}(k, d, w),
\end{aligned}
$$

for all $t, s, u, v, w>0$ and $c \neq e, e \neq f, f \neq g, g \neq k, k \neq d$.

Definition 1 ([4]). Let $*:[0,1] \times[0,1] \rightarrow[0,1]$ be a binary operation, then $*$ is said to be continuous triangular norm (in short, continuous $t$-norm), if, for all $c_{1}, c_{2}, c_{3}, c_{4} \in[0,1]$, the

1. $*\left(c_{1}, c_{2}\right)=*\left(c_{2}, c_{1}\right)$;

2. $*\left(c_{1}, *\left(c_{2}, c_{3}\right)\right)=*\left(*\left(c_{1}, c_{2}\right), c_{3}\right)$;

3. $*$ is continuous;

4. $*\left(c_{1}, c_{2}\right) \leq *\left(c_{3}, c_{4}\right)$ whenever $c_{1} \leq c_{3}, c_{2} \leq c_{4}$.

5. $*(1, c)=c$ for any $c \in[0,1]$.

The following definitions are the fuzzy forms of $b$-metric spaces [10], rectangular $b$-metric spaces [17], extended $b$-metric spaces [11], and extended rectangular $b$-metric spaces [28] that will be utilized to characterize our main concept.

Definition 2 ([29]). Let $X$ be a nonempty set, $b \geq 1$ a real number, $*$ a continuous $t$-norm, and $m$ be a fuzzy set on $X \times X \times[0, \infty)$. Then, $m$ is said to be a fuzzy b-metric on $X$, if, for all $c, d, e \in X$, $m$ fulfills the following criteria:

1. $m(c, d, 0)=0$ for $t=0$;

2. $m(c, d, t)=1$ if and only if $c=d$ for all $t>0$;

3. $m(c, d, t)=m(d, c, t)$;

4. $\quad m(c, d, b(t+s)) \geq m(c, e, t) * m(e, d, s)$ for all $s, t>0$;

5. $m(c, d,):.(0, \infty) \rightarrow[0,1]$ is left continuous and $\lim _{t \rightarrow \infty} m(c, d, t)=1$.

The quadruple $(X, m, *, b)$ is called fuzzy b-metric space.

Definition 3 ([12]). Let $X$ be a nonempty set, $b: X \times X \rightarrow[1, \infty)$, * a continuous $t$-norm, and $m$ be a fuzzy set on $X \times X \times[0, \infty)$. Then, $m$ is said to be an extended fuzzy b-metric on $X$, if, for all $c, d, e \in X, m$ fulfills the following criteria:

1. $m(c, d, 0)=0$ for $t=0$;

2. $m(c, d, t)=1$ if and only if $c=d$ for all $t>0$;

3. $m(c, d, t)=m(d, c, t)$;

4. $\quad m(c, d, b(c, d)(t+s)) \geq m(c, e, t) * m(e, d, s)$ for all $s, t>0$;

5. $m(c, d,):.(0, \infty) \rightarrow[0,1]$ is left continuous.

The quadruple $(X, m, *, b(c, d))$ is called an extended fuzzy b-metric space.

Definition 4 ([13]). Let $X$ be a nonempty set, $b \geq 1$ a real number, $*$ a continuous $t$-norm, and $m$ be a fuzzy set on $X \times X \times[0, \infty)$. Then, $m$ is called fuzzy rectangular $b$-metric, if, for any $c, d \in X$ and all distinct points $e, f \in X \backslash\{c, d\}$, the following requirements are fulfilled:

1. $m(c, d, 0)=0$ for $t=0$;

2. $m(c, d, t)=1$ if and only if $c=d$ for all $t>0$;

3. $m(c, d, t)=m(d, c, t)$;

4. $\quad m(c, d, b(t+s+u)) \geq m(c, e, t) * m(e, f, s) * m(f, d, u)$ for all $t, s, u>0$;

5. $m(c, d,):.(0, \infty) \rightarrow[0,1]$ is left continuous and $\lim _{t \rightarrow \infty} m(c, d, t)=1$.

Then, $(X, m, *, b)$ is known as a fuzzy rectangular b-metric space.

Definition 5 ([30]). Let $*$ a continuous $t$-norm, $b: X \times X \rightarrow[1, \infty)$ be a given function and $m$ be a fuzzy set on $X \times X \times[0, \infty)$ satisfying the following conditions, for all $c, d \in X$ and all distinct points $e, f \in X \backslash\{c, d\}$ : 
1. $m(c, d, 0)=0$ for $t=0$;

2. $m(c, d, t)=1$ if and only if $c=d$ for all $t>0$;

3. $m(c, d, t)=m(d, c, t)$;

4. $\quad m(c, d, b(c, d)(t+s+u)) \geq m(c, e, t) * m(e, f, s) * m(f, d, u)$ for all $t, s, u>0$;

5. $m(c, d,):.(0, \infty) \rightarrow[0,1]$ is left continuous and $\lim _{t \rightarrow \infty} m(c, d, t)=1$.

Then, $(X, m, *, b(c, d))$ is called an extended fuzzy rectangular b-metric space.

Kalpana et al. [21] recently proposed the notion of an extended hexagonal $b$-metric spaces.

Definition 6. Let $X$ be a non-empty set and $b: X \times X \rightarrow[1, \infty)$. A function $m: X \times X \rightarrow[0, \infty)$ is called an extended hexagonal $b$-metric if it satisfies:

1. $m(c, d)=0 \Leftrightarrow c=d$ for all $c, d \in X$;

2. $m(c, d)=m(d, c)$ for all $c, d \in X$;

3. $m(c, d) \leq b(c, d)[m(c, e)+m(e, f)+m(f, g)+m(g, h)+m(h, d)]$ for all $c, d, e, f, g, h \in$ $X$ and $c \neq e, e \neq f, f \neq g, g \neq h, h \neq d$;

The pair $(X, m)$ is called an extended hexagonal b-metric space.

The rest of this article is laid out as follows:

- $\quad$ Section 2 presents a new generalization of FMS, namely, fuzzy extended hexagonal $b$-metric spaces, by first providing essential concepts and ideas used in exploring the outcomes of this research. Following that, in this section, an example is provided with a focus on the fuzzy extended hexagonal $b$-metric spaces and explores the notion of convergence sequence, Cauchy sequence and completness in FEHb-MS, relying on certain topological features of the examined space.

- In Section 3, by adding additional conditions to the functions that offer Banach contraction and fuzzy $\psi$-contraction in FEHb-MS, we established new fixed point results in this study.

- Eventually, in Section 4, we examine the existence and uniqueness of solutions for nonlinear fractional differential equations in the sense of Caputo derivative applying the fixed point results reported in the preceding section.

\section{Main Results}

This section begins with an introductory of fuzzy extended hexagonal $b$-metric spaces (or simply FEHb-MS), as well as an example of the space defined.

Definition 7. Let $X \neq \varnothing, b: X \times X \rightarrow[1, \infty), *$ a continuous $t$-norm, and $m$ be a fuzzy set on $X \times X \times[0, \infty)$. Then, $m$ is called fuzzy extended hexagonal $b$-metric, if, for all $c, d, e, f, g, k \in X$ and $c \neq e, e \neq f, f \neq g, g \neq k, k \neq d$, the following conditions are satisfied:

$\left[\mathfrak{m}_{\mathrm{h}} 1\right] \mathfrak{m}_{\mathrm{h}}(c, d, 0)=0$ for $t=0$;

$\left[\mathfrak{m}_{\mathrm{h}} 2\right] \mathfrak{m}_{\mathrm{h}}(c, d, t)=1, \forall t>0$ if and only if $c=d$;

$\left[\mathfrak{m}_{\mathrm{h}} 3\right] \mathfrak{m}_{\mathrm{h}}(c, d, t)=\mathfrak{m}_{\mathrm{h}}(d, c, t)$;

$\left[\mathfrak{m}_{\mathrm{h}} 4\right] \mathfrak{m}_{\mathrm{h}}(c, d, b(c, d)(t+s+u+v+w)) \geq \mathfrak{m}_{\mathrm{h}}(c, e, t) * \mathfrak{m}_{\mathrm{h}}(e, f, s) * \mathfrak{m}_{\mathrm{h}}(f, g, u) * \mathfrak{m}_{\mathrm{h}}(g, k, v) *$ $\mathfrak{m}_{\mathrm{h}}(k, d, w)$ for all $t, s, u, v, w>0$;

$\left[\mathfrak{m}_{\mathrm{h}} 5\right] \mathfrak{m}_{\mathrm{h}}(c, d,):.(0, \infty) \rightarrow[0,1]$ is left continuous.

Then, $\left(X, \mathfrak{m}_{\mathrm{h}}, *\right)$ is known as fuzzy extended hexagonal b-metric space.

Example 1. Let $X=\{1,2,3,4,5,6\}$ and functions $b: X \times X \rightarrow[1, \infty)$ and $h_{v}: X \times X \rightarrow \mathbb{R}$ such that $h_{v}$ is symmetric can be defined as:

$b(c, d)=c+d, \forall c, d \in X$,

$h_{v}(c, d)=0, \forall c=d$; 
$h_{v}(1,2)=700 ; h_{v}(1, c)=h_{v}(2, c)=50, \forall c \in X \backslash\{1,2,6\} ;$

$h_{v}(3,4)=h_{v}(3,5)=h_{v}(4,5)=50$;

$h_{v}(c, 6)=150, \forall c \in X \backslash\{6\}$.

Then, it is immediately evident that $\left(X, h_{v}\right)$ is an extended hexagonal b-metric space. Let $\mathfrak{m}_{\mathrm{h}}: X \times X \times[0, \infty) \rightarrow[0,1]$ be specified in the following form:

$$
\mathfrak{m}_{\mathrm{h}}(c, d, t)= \begin{cases}\frac{\alpha t}{\alpha t+\gamma h_{v}(c, d)}, & \text { if } t>0 \\ 0, & \text { if } t=0\end{cases}
$$

where $\alpha, \gamma$ are positive real numbers and $t$-norm is defined by $t_{1} * t_{2}=\min \left\{t_{1}, t_{2}\right\} .\left(X, \mathfrak{m}_{\mathrm{h}}, *\right)$ is therefore shown to be a fuzzy extended hexagonal b-metric space. We observe that the criteria $\left[\mathfrak{m}_{\mathrm{h}} 1\right],\left[\mathfrak{m}_{\mathrm{h}} 2\right],\left[\mathfrak{m}_{\mathrm{h}} 3\right]$ and $\left[\mathfrak{m}_{\mathrm{h}} 5\right]$ of Definition 7 are provably true. To demonstrate the property $\left[\mathfrak{m}_{\mathrm{h}} 4\right]$ for all $c, d \in X$, consider the following

$$
\mathfrak{m}_{\mathrm{h}}(c, d, b(c, d)(t+s+u+v+w))=\frac{\alpha b(c, d)(t+s+u+v+w)}{\alpha b(c, d)(t+s+u+v+w)+\gamma h_{v}(c, d)} .
$$

For $c=1, d=4$,

$$
\begin{aligned}
& \mathfrak{m}_{\mathrm{h}}(1,4, b(1,4)(t+s+u+v+w))=\frac{\alpha b(1,4)(t+s+u+v+w)}{\alpha b(1,4)(t+s+u+v+w)+\gamma h_{v}(1,4)} \\
&=\frac{5 \alpha(t+s+u+v+w)}{5 \alpha(t+s+u+v+w)+50 \gamma} \\
&=1-\frac{50 \gamma}{5 \alpha(t+s+u+v+w)+50 \gamma} \\
& \mathfrak{m}_{\mathrm{h}}(1,2, t)=\frac{\alpha t}{\alpha t+700 \gamma}=1-\frac{700 \gamma}{\alpha t+700 \gamma} ; \quad \mathfrak{m}_{\mathrm{h}}(2,3, s)=\frac{\alpha s}{\alpha s+50 \gamma}=1-\frac{50 \gamma}{\alpha s+50 \gamma} ; \\
& \mathfrak{m}_{\mathrm{h}}(3,5, u)=\frac{\alpha u}{\alpha u+50 \gamma}=1-\frac{50 \gamma}{\alpha u+50 \gamma} ; \quad \mathfrak{m}_{\mathrm{h}}(5,6, v)=\frac{\alpha v}{\alpha v+150 \gamma}=1-\frac{150 \gamma}{\alpha v+150 \gamma} ; \\
& \mathfrak{m}_{\mathrm{h}}(6,4, w)=\frac{\alpha w}{\alpha w+150 \gamma}=1-\frac{150 \gamma}{\alpha w+150 \gamma} .
\end{aligned}
$$

As a result, for all $t, s, w, u, v>0$, we observe that

$$
\begin{aligned}
\mathfrak{m}_{\mathrm{h}}(1,4, b(1,4)(t+s+u+v+w)) & =1-\frac{50 \gamma}{5 \alpha(t+s+u+v+w)+50 \gamma} \\
& =1-\frac{700 \gamma}{70 \alpha(t+s+u+v+w)+700 \gamma} \\
& >1-\frac{700 \gamma}{70 \alpha t+700 \gamma} \\
& >1-\frac{700 \gamma}{\alpha t+700 \gamma}=\mathfrak{m}_{\mathrm{h}}(1,2, t) .
\end{aligned}
$$

It can further be demonstrated that

$$
\begin{aligned}
& \mathfrak{m}_{\mathrm{h}}(1,4, b(1,4)(t+s+u+v+w))>\mathfrak{m}_{\mathrm{h}}(2,3, t), \mathfrak{m}_{\mathrm{h}}(1,4, b(1,4)(t+s+u+v+w))>\mathfrak{m}_{\mathrm{h}}(3,5, t), \\
& \mathfrak{m}_{\mathrm{h}}(1,4, b(1,4)(t+s+u+v+w))>\mathfrak{m}_{\mathrm{h}}(5,6, t), \mathfrak{m}_{\mathrm{h}}(1,4, b(1,4)(t+s+u+v+w))>\mathfrak{m}_{\mathrm{h}}(6,4, t) .
\end{aligned}
$$

Consequently, it implies that

$\mathfrak{m}_{\mathrm{h}}(1,4, b(1,4)(t+s+u+v+w)) \geq \min \left\{\mathfrak{m}_{\mathrm{h}}(1,2, t), \mathfrak{m}_{\mathrm{h}}(2,3, t), \mathfrak{m}_{\mathrm{h}}(3,5, t), \mathfrak{m}_{\mathrm{h}}(5,6, t), \mathfrak{m}_{\mathrm{h}}(6,4, t)\right\}$. 
Hence,

$\mathfrak{m}_{\mathrm{h}}(1,4, b(1,4)(t+s+u+v+w)) \geq \mathfrak{m}_{\mathrm{h}}(1,2, t) * \mathfrak{m}_{\mathrm{h}}(2,3, t) * \mathfrak{m}_{\mathrm{h}}(3,5, t) * \mathfrak{m}_{\mathrm{h}}(5,6, t) * \mathfrak{m}_{\mathrm{h}}(6,4, t)$.

We can verify the remaining cases in the same way. Thus, for all $t, s, u, v, w>0$, $\mathfrak{m}_{\mathrm{h}}(c, d, b(c, d)(t+s+u+v+w)) \geq \mathfrak{m}_{\mathrm{h}}(c, e, t) * \mathfrak{m}_{\mathrm{h}}(e, f, s) * \mathfrak{m}_{\mathrm{h}}(f, g, u) * \mathfrak{m}_{\mathrm{h}}(g, k, v) * \mathfrak{m}_{\mathrm{h}}(k, d, w)$.

Therefore, $\left(X, \mathfrak{m}_{\mathrm{h}}, *\right)$ is a fuzzy extended hexagonal extended $b$-metric space.

Definition 8. Let $\left(X, \mathfrak{m}_{\mathrm{h}}, *\right)$ be a fuzzy extended hexagonal b-metric space. A sequence $\left\{c_{n}\right\}$ in $X$ is said to be

- convergent, if, for all $t>0$, there exists $c$ in $X$ such that

$$
\lim _{n \rightarrow \infty} \mathfrak{m}_{\mathrm{h}}\left(c_{n}, c, t\right)=1
$$

- $\quad$ Cauchy if and only if for all $t>0$ and for each $\epsilon>0$, there exists $n_{0} \in \mathbb{N}$ such that

$$
\mathfrak{m}_{\mathrm{h}}\left(c_{n}, c_{m}, t\right) \geq 1-\epsilon, \text { for all } m, n \geq n_{0} .
$$

- $\quad\left(X, \mathfrak{m}_{\mathrm{h}}, *\right)$ is called complete FEHb-MS, if every Cauchy sequence in $X$ converges to some point $c$ in $X$.

Definition 9. Let $\left(X, \mathfrak{m}_{\mathrm{h}}, *\right)$ be a fuzzy extended hexagonal $b$-metric space. For $c \in X, r \in(0,1)$ and $t>0$, we define the open ball

$$
B(c, r, t)=\left\{d \in X: \mathfrak{m}_{\mathrm{h}}(c, d, t)>1-r\right\} .
$$

The corresponding topology is defined as

$$
\tau_{\mathfrak{m}_{\mathrm{h}}}=\{Y \subset X: \forall c \in Y \exists r \in(0,1) \text { and } t>0, B(c, r, t) \subset Y\} .
$$

\section{Fixed Point Results on FEHb-MS}

Theorem 1. Let $\left(X, \mathfrak{m}_{\mathrm{h}}, *\right)$ be a complete fuzzy extended hexagonal $b$-metric space and $b: X \times$ $X \rightarrow[1, \infty)$ such that

$$
\lim _{t \rightarrow \infty} \mathfrak{m}_{\mathrm{h}}(c, d, t)=1, \forall c, d \in X \text { and } t>0 .
$$

Let $F: X \rightarrow X$ be a mapping that fulfills the criteria

$$
\mathfrak{m}_{\mathrm{h}}(F c, F d, \beta t) \geq \mathfrak{m}_{\mathrm{h}}(c, d, t), \forall c, d \in X \text { and } t>0,
$$

where $\beta \in(0,1)$. Furthermore, if, for $c_{0} \in X$ and $n, q \in \mathbb{N}$, it holds $b\left(c_{n}, c_{n+q}\right)<\frac{1}{\beta}$ where $c_{n}=F^{n} c_{0}$, then $F$ has a unique fixed point.

Proof. Let $c_{0} \in X$ and construct a sequence $\left\{c_{n}\right\}$ by

$$
c_{n}=F c_{n-1} \text { for all } n \in \mathbb{N} \text {. }
$$

Without losing generality, assume that $c_{n} \neq c_{n+1}, \forall n \geq 0$. According to (6), we have 


$$
\begin{aligned}
& \mathfrak{m}_{\mathrm{h}}\left(c_{n}, c_{n+1}, t\right)= \mathfrak{m}_{\mathrm{h}}\left(F c_{n-1}, F c_{n}, t\right) \\
& \geq \mathfrak{m}_{\mathrm{h}}\left(c_{n-1}, c_{n}, \frac{t}{\beta}\right) \\
& \vdots \\
& \geq \mathfrak{m}_{\mathrm{h}}\left(c_{0}, c_{1}, \frac{t}{\beta^{n}}\right) .
\end{aligned}
$$

It follows in a similar manner that

$$
\begin{aligned}
& \mathfrak{m}_{\mathrm{h}}\left(c_{n}, c_{n+2}, t\right) \geq \mathfrak{m}_{\mathrm{h}}\left(c_{0}, c_{2}, \frac{t}{\beta^{n}}\right), \\
& \mathfrak{m}_{\mathrm{h}}\left(c_{n}, c_{n+3}, t\right) \geq \mathfrak{m}_{\mathrm{h}}\left(c_{0}, c_{3}, \frac{t}{\beta^{n}}\right),
\end{aligned}
$$

and

$$
\mathfrak{m}_{\mathrm{h}}\left(c_{n}, c_{n+4}, t\right) \geq \mathfrak{m}_{\mathrm{h}}\left(c_{0}, c_{4}, \frac{t}{\beta^{n}}\right) .
$$

It therefore implies that, if $m=1,2, \ldots$,

$$
\begin{aligned}
& \mathfrak{m}_{\mathrm{h}}\left(c_{n}, c_{n+4 m+1}, t\right) \geq \mathfrak{m}_{\mathrm{h}}\left(c_{0}, c_{4 m+1}, \frac{t}{\beta^{n}}\right), \\
& \mathfrak{m}_{\mathrm{h}}\left(c_{n}, c_{n+4 m+2}, t\right) \geq \mathfrak{m}_{\mathrm{h}}\left(c_{0}, c_{4 m+2}, \frac{t}{\beta^{n}}\right), \\
& \mathfrak{m}_{\mathrm{h}}\left(c_{n}, c_{n+4 m+3}, t\right) \geq \mathfrak{m}_{\mathrm{h}}\left(c_{0}, c_{4 m+3}, \frac{t}{\beta^{n}}\right),
\end{aligned}
$$

and

$$
\mathfrak{m}_{\mathrm{h}}\left(c_{n}, c_{n+4 m+4}, t\right) \geq \mathfrak{m}_{\mathrm{h}}\left(c_{0}, c_{4 m+4}, \frac{t}{\beta^{n}}\right) .
$$

Expressing $t=t / 5+t / 5+t / 5+t / 5+t / 5$ and by employing $(7)$ and $\left(\mathfrak{m}_{\mathrm{h}} 4\right)$, we get

$$
\begin{aligned}
\mathfrak{m}_{\mathrm{h}}\left(c_{0}, c_{5}, t\right) \geq & \mathfrak{m}_{\mathrm{h}}\left(c_{0}, c_{1}, \frac{t / 5}{b\left(c_{0}, c_{5}\right)}\right) * \mathfrak{m}_{\mathrm{h}}\left(c_{1}, c_{2}, \frac{t / 5}{b\left(c_{0}, c_{5}\right)}\right) * \mathfrak{m}_{\mathrm{h}}\left(c_{2}, c_{3}, \frac{t / 5}{b\left(c_{0}, c_{5}\right)}\right) \\
& * \mathfrak{m}_{\mathrm{h}}\left(c_{3}, c_{4}, \frac{t / 5}{b\left(c_{0}, c_{5}\right)}\right) * \mathfrak{m}_{\mathrm{h}}\left(c_{4}, c_{5}, \frac{t / 5}{b\left(c_{0}, c_{5}\right)}\right) \\
\geq & \mathfrak{m}_{\mathrm{h}}\left(c_{0}, c_{1}, \frac{t / 5}{b\left(c_{0}, c_{5}\right)}\right) * \mathfrak{m}_{\mathrm{h}}\left(c_{0}, c_{1}, \frac{t / 5}{\beta b\left(c_{0}, c_{5}\right)}\right) * \mathfrak{m}_{\mathrm{h}}\left(c_{0}, c_{1}, \frac{t / 5}{\beta^{2} b\left(c_{0}, c_{5}\right)}\right) \\
& * \mathfrak{m}_{\mathrm{h}}\left(c_{0}, c_{1}, \frac{t / 5}{\beta^{3} b\left(c_{0}, c_{5}\right)}\right) * \mathfrak{m}_{\mathrm{h}}\left(c_{0}, c_{1}, \frac{t / 5}{\beta^{4} b\left(c_{0}, c_{5}\right)}\right) .
\end{aligned}
$$


With a similar approach, one can obtain

$$
\begin{aligned}
\mathfrak{m}_{\mathrm{h}}\left(c_{0}, c_{9}, t\right) \geq & \mathfrak{m}_{\mathrm{h}}\left(c_{0}, c_{1}, \frac{t / 5}{b\left(c_{0}, c_{9}\right)}\right) * \mathfrak{m}_{\mathrm{h}}\left(c_{1}, c_{2}, \frac{t / 5}{b\left(c_{0}, c_{9}\right)}\right) * \mathfrak{m}_{\mathrm{h}}\left(c_{2}, c_{3}, \frac{t / 5}{b\left(c_{0}, c_{9}\right)}\right) \\
& * \mathfrak{m}_{\mathrm{h}}\left(c_{3}, c_{4}, \frac{t / 5}{b\left(c_{0}, c_{9}\right)}\right) * \mathfrak{m}_{\mathrm{h}}\left(c_{4}, c_{9}, \frac{t / 5}{b\left(c_{0}, c_{9}\right)}\right) \\
\geq & \mathfrak{m}_{\mathrm{h}}\left(c_{0}, c_{1}, \frac{t / 5}{b\left(c_{0}, c_{9}\right)}\right) * \mathfrak{m}_{\mathrm{h}}\left(c_{1}, c_{2}, \frac{t / 5}{b\left(c_{0}, c_{9}\right)}\right) * \mathfrak{m}_{\mathrm{h}}\left(c_{2}, c_{3}, \frac{t / 5}{b\left(c_{0}, c_{9}\right)}\right) \\
& * \mathfrak{m}_{\mathrm{h}}\left(c_{3}, c_{4}, \frac{t / 5}{b\left(c_{0}, c_{9}\right)}\right) * \mathfrak{m}_{\mathrm{h}}\left(c_{4}, c_{5}, \frac{t / 5^{2}}{b\left(c_{0}, c_{9}\right) b\left(c_{4}, c_{9}\right)}\right) \\
& * \mathfrak{m}_{\mathrm{h}}\left(c_{5}, c_{6}, \frac{t / 5^{2}}{b\left(c_{0}, c_{9}\right) b\left(c_{4}, c_{9}\right)}\right) * \mathfrak{m}_{\mathrm{h}}\left(c_{6}, c_{7}, \frac{t / 5^{2}}{b\left(c_{0}, c_{9}\right) b\left(c_{4}, c_{9}\right)}\right) \\
& * \mathfrak{m}_{\mathrm{h}}\left(c_{7}, c_{8}, \frac{t / 5^{2}}{b\left(c_{0}, c_{9}\right) b\left(c_{4}, c_{9}\right)}\right) * \mathfrak{m}_{\mathrm{h}}\left(c_{8}, c_{9}, \frac{t / 5^{2}}{b\left(c_{0}, c_{9}\right) b\left(c_{4}, c_{9}\right)}\right) \\
\geq & \mathfrak{m}_{\mathrm{h}}\left(c_{0}, c_{1}, \frac{t / 5}{b\left(c_{0}, c_{9}\right)}\right) * \mathfrak{m}_{\mathrm{h}}\left(c_{0}, c_{1}, \frac{t / 5}{\beta b\left(c_{0}, c_{9}\right)}\right) * \mathfrak{m}_{\mathrm{h}}\left(c_{0}, c_{1}, \frac{t / 5}{\beta^{2} b\left(c_{0}, c_{9}\right)}\right) \\
& * \mathfrak{m}_{\mathrm{h}}\left(c_{0}, c_{1}, \frac{t / 5}{\beta^{3} b\left(c_{0}, c_{9}\right)}\right) * \mathfrak{m}_{\mathrm{h}}\left(c_{0}, c_{1}, \frac{t / 5^{2}}{\beta^{4} b\left(c_{0}, c_{9}\right) b\left(c_{4}, c_{9}\right)}\right) \\
& * \mathfrak{m}_{\mathrm{h}}\left(c_{0}, c_{1}, \frac{t / 5^{2}}{\beta^{5} b\left(c_{0}, c_{9}\right) b\left(c_{4}, c_{9}\right)}\right) * \mathfrak{m}_{\mathrm{h}}\left(c_{0}, c_{1}, \frac{t / 5^{2}}{\beta^{6} b\left(c_{0}, c_{9}\right) b\left(c_{4}, c_{9}\right)}\right) \\
& * \mathfrak{m}_{\mathrm{h}}\left(c_{0}, c_{1}, \frac{t / 5^{2}}{\beta^{7} b\left(c_{0}, c_{9}\right) b\left(c_{4}, c_{9}\right)}\right) * \mathfrak{m}_{\mathrm{h}}\left(c_{0}, c_{1}, \frac{t / 5^{2}}{\beta^{8} b\left(c_{0}, c_{9}\right) b\left(c_{4}, c_{9}\right)}\right) .
\end{aligned}
$$

Through induction, we get for each $m=1,2,3 \ldots$

$$
\begin{aligned}
\mathfrak{m}_{\mathrm{h}}\left(c_{0}, c_{4 m+1}, t\right) \geq & {\left[*_{i=0}^{m-1} \mathfrak{m}_{\mathrm{h}}\left(c_{0}, c_{1}, \frac{t / 5^{i+1}}{\beta^{4 i} \prod_{j=0}^{i} b\left(c_{4 j}, c_{4 m+1}\right)}\right)\right] *\left[*_{i=0}^{m-1} \mathfrak{m}_{\mathrm{h}}\left(c_{0}, c_{1}, \frac{t / 5^{i+1}}{\beta^{4 i+1} \prod_{j=0}^{i} b\left(c_{4 j}, c_{4 m+1}\right)}\right)\right] } \\
& *\left[*_{i=0}^{m-1} \mathfrak{m}_{\mathrm{h}}\left(c_{0}, c_{1}, \frac{t / 5^{i+1}}{\beta^{4 i+2} \prod_{j=0}^{i} b\left(c_{4 j}, c_{4 m+1}\right)}\right)\right] *\left[*_{i=0}^{m-1} \mathfrak{m}_{\mathrm{h}}\left(c_{0}, c_{1}, \frac{t / 5^{i+1}}{\beta^{4 i+3} \prod_{j=0}^{i} b\left(c_{4 j}, c_{4 m+1}\right)}\right)\right] \\
& * \mathfrak{m}_{\mathrm{h}}\left(c_{0}, c_{1}, \frac{t / 5^{m}}{\beta^{4 m} \prod_{j=0}^{m-1} b\left(c_{4 j}, c_{4 m+1}\right)}\right) .
\end{aligned}
$$

Now, using the aforementioned inequality, we can obtain from (11) that

$$
\begin{aligned}
\mathfrak{m}_{\mathrm{h}}\left(c_{n}, c_{n+4 m+1}, t\right) & \geq \mathfrak{m}_{\mathrm{h}}\left(c_{0}, c_{4 m+1}, \frac{t}{\beta^{n}}\right) \\
\geq & {\left[*_{i=0}^{m-1} \mathfrak{m}_{\mathrm{h}}\left(c_{0}, c_{1}, \frac{t / 5^{i+1}}{\beta^{n+4 i} \prod_{j=0}^{i} b\left(c_{4 j}, c_{4 m+1}\right)}\right)\right] *\left[*_{i=0}^{m-1} \mathfrak{m}_{\mathrm{h}}\left(c_{0}, c_{1}, \frac{t / 5^{i+1}}{\beta^{n+4 i+1} \prod_{j=0}^{i} b\left(c_{4 j}, c_{4 m+1}\right)}\right)\right] }
\end{aligned}
$$


Symmetry 2021, 13, 2032

8 of 22

$$
\begin{aligned}
& *\left[*_{i=0}^{m-1} \mathfrak{m}_{\mathrm{h}}\left(c_{0}, c_{1}, \frac{t / 5^{i+1}}{\beta^{n+4 i+2} \prod_{j=0}^{i} b\left(c_{4 j}, c_{4 m+1}\right)}\right)\right] *\left[*_{i=0}^{m-1} \mathfrak{m}_{\mathrm{h}}\left(c_{0}, c_{1}, \frac{t / 5^{i+1}}{\beta^{n+4 i+3} \prod_{j=0}^{i} b\left(c_{4 j}, c_{4 m+1}\right)}\right)\right] \\
& * \mathfrak{m}_{\mathrm{h}}\left(c_{0}, c_{1}, \frac{t / 5^{m}}{\beta^{n+4 m} \prod_{j=0}^{m-1} b\left(c_{4 j}, c_{4 m+1}\right)}\right)
\end{aligned}
$$

Furthermore, from (7) and (8) as well as the hexagonal property, we deduce

$$
\begin{aligned}
\mathfrak{m}_{\mathrm{h}}\left(c_{0}, c_{6}, t\right) \geq & \mathfrak{m}_{\mathrm{h}}\left(c_{0}, c_{1}, \frac{t / 5}{b\left(c_{0}, c_{6}\right)}\right) * \mathfrak{m}_{\mathrm{h}}\left(c_{1}, c_{2}, \frac{t / 5}{b\left(c_{0}, c_{6}\right)}\right) * \mathfrak{m}_{\mathrm{h}}\left(c_{2}, c_{3}, \frac{t / 5}{b\left(c_{0}, c_{6}\right)}\right) \\
& * \mathfrak{m}_{\mathrm{h}}\left(c_{3}, c_{4}, \frac{t / 5}{b\left(c_{0}, c_{6}\right)}\right) * \mathfrak{m}_{\mathrm{h}}\left(c_{4}, c_{6}, \frac{t / 5}{b\left(c_{0}, c_{6}\right)}\right) \\
\geq & \mathfrak{m}_{\mathrm{h}}\left(c_{0}, c_{1}, \frac{t / 5}{b\left(c_{0}, c_{6}\right)}\right) * \mathfrak{m}_{\mathrm{h}}\left(c_{0}, c_{1}, \frac{t / 5}{\beta b\left(c_{0}, c_{6}\right)}\right) * \mathfrak{m}_{\mathrm{h}}\left(c_{0}, c_{1}, \frac{t / 5}{\beta^{2} b\left(c_{0}, c_{6}\right)}\right) \\
& * \mathfrak{m}_{\mathrm{h}}\left(c_{0}, c_{1}, \frac{t / 5}{\beta^{3} b\left(c_{0}, c_{6}\right)}\right) * \mathfrak{m}_{\mathrm{h}}\left(c_{0}, c_{2}, \frac{t / 5}{\beta^{4} b\left(c_{0}, c_{6}\right)}\right)
\end{aligned}
$$

and

$$
\begin{aligned}
\mathfrak{m}_{\mathrm{h}}\left(c_{0}, c_{10}, t\right) \geq & \mathfrak{m}_{\mathrm{h}}\left(c_{0}, c_{1}, \frac{t / 5}{b\left(c_{0}, c_{10}\right)}\right) * \mathfrak{m}_{\mathrm{h}}\left(c_{1}, c_{2}, \frac{t / 5}{b\left(c_{0}, c_{10}\right)}\right) * \mathfrak{m}_{\mathrm{h}}\left(c_{2}, c_{3}, \frac{t / 5}{b\left(c_{0}, c_{10}\right)}\right) \\
& * \mathfrak{m}_{\mathrm{h}}\left(c_{3}, c_{4}, \frac{t / 5}{b\left(c_{0}, c_{10}\right)}\right) * \mathfrak{m}_{\mathrm{h}}\left(c_{4}, c_{10}, \frac{t / 5}{b\left(c_{0}, c_{10}\right)}\right) \\
\geq & \mathfrak{m}_{\mathrm{h}}\left(c_{0}, c_{1}, \frac{t / 5}{b\left(c_{0}, c_{10}\right)}\right) * \mathfrak{m}_{\mathrm{h}}\left(c_{1}, c_{2}, \frac{t / 5}{b\left(c_{0}, c_{10}\right)}\right) * \mathfrak{m}_{\mathrm{h}}\left(c_{2}, c_{3}, \frac{t / 5}{b\left(c_{0}, c_{10}\right)}\right) \\
& * \mathfrak{m}_{\mathrm{h}}\left(c_{3}, c_{4}, \frac{t / 5}{b\left(c_{0}, c_{10}\right)}\right) * \mathfrak{m}_{\mathrm{h}}\left(c_{4}, c_{5}, \frac{t / 5^{2}}{b\left(c_{0}, c_{10}\right) b\left(c_{4}, c_{10}\right)}\right) \\
& * \mathfrak{m}_{\mathrm{h}}\left(c_{5}, c_{6}, \frac{t / 5^{2}}{b\left(c_{0}, c_{10}\right) b\left(c_{4}, c_{10}\right)}\right) * \mathfrak{m}_{\mathrm{h}}\left(c_{6}, c_{7}, \frac{t / 5^{2}}{b\left(c_{0}, c_{10}\right) b\left(c_{4}, c_{10}\right)}\right) \\
& * \mathfrak{m}_{\mathrm{h}}\left(c_{7}, c_{8}, \frac{t / 5^{2}}{b\left(c_{0}, c_{10}\right) b\left(c_{4}, c_{10}\right)}\right) * \mathfrak{m}_{\mathrm{h}}\left(c_{8}, c_{10}, \frac{t / 5^{2}}{b\left(c_{0}, c_{10}\right) b\left(c_{4}, c_{10}\right)}\right) \\
\geq & \left.*_{i=0}^{3} \mathfrak{m}_{\mathrm{h}}\left(c_{0}, c_{1}, \frac{t / 5}{\beta^{i} b\left(c_{0}, c_{10}\right)}\right)\right] *\left[*_{i=4}^{7} \mathfrak{m}_{\mathrm{h}}\left(c_{0}, c_{1}, \frac{t / 5}{\beta^{i} b\left(c_{0}, c_{10}\right) b\left(c_{0}, c_{10}\right)}\right)\right] \\
& * \mathfrak{m}_{\mathrm{h}}\left(c_{0}, c_{2}, \frac{t / 5}{\beta^{8} b\left(c_{0}, c_{10}\right) b\left(c_{0}, c_{10}\right)}\right) .
\end{aligned}
$$

Through induction, we get the following inequality for each $m=1,2,3 \ldots$

$$
\mathfrak{m}_{\mathrm{h}}\left(c_{0}, c_{4 m+2}, t\right) \geq\left[*_{i=0}^{m-1} \mathfrak{m}_{\mathrm{h}}\left(c_{0}, c_{1}, \frac{t / 5^{i+1}}{\beta^{4 i} \prod_{j=0}^{i} b\left(c_{4 j}, c_{4 m+2}\right)}\right)\right] *\left[*_{i=0}^{m-1} \mathfrak{m}_{\mathrm{h}}\left(c_{0}, c_{1}, \frac{t / 5^{i+1}}{\beta^{4 i+1} \prod_{j=0}^{i} b\left(c_{4 j}, c_{4 m+2}\right)}\right)\right]
$$




$$
\begin{aligned}
& *\left[*_{i=0}^{m-1} \mathfrak{m}_{\mathrm{h}}\left(c_{0}, c_{1}, \frac{t / 5^{i+1}}{\beta^{4 i+2} \prod_{j=0}^{i} b\left(c_{4 j}, c_{4 m+2}\right)}\right)\right] *\left[*_{i=0}^{m-1} \mathfrak{m}_{\mathrm{h}}\left(c_{0}, c_{1}, \frac{t / 5^{i+1}}{\beta^{4 i+3} \prod_{j=0}^{i} b\left(c_{4 j}, c_{4 m+2}\right)}\right)\right] \\
& \quad * \mathfrak{m}_{\mathrm{h}}\left(c_{0}, c_{2}, \frac{t / 5^{m}}{\beta^{4 m} \prod_{j=0}^{m-1} b\left(c_{4 j}, c_{4 m+2}\right)}\right) .
\end{aligned}
$$

By using the preceding inequality in (12), we get

$$
\begin{aligned}
& \mathfrak{m}_{\mathrm{h}}\left(c_{n}, c_{n+4 m+2}, t\right) \geq \mathfrak{m}_{\mathrm{h}}\left(c_{0}, c_{4 m+2}, \frac{t}{\beta^{n}}\right) \\
& \geq {\left[*_{i=0}^{m-1} \mathfrak{m}_{\mathrm{h}}\left(c_{0}, c_{1}, \frac{t / 5^{i+1}}{\beta^{n+4 i} \prod_{j=0}^{i} b\left(c_{4 j}, c_{4 m+2}\right)}\right)\right] *\left[*_{i=0}^{m-1} \mathfrak{m}_{\mathrm{h}}\left(c_{0}, c_{1}, \frac{t / 5^{i+1}}{\beta^{n+4 i+1} \prod_{j=0}^{i} b\left(c_{4 j}, c_{4 m+2}\right)}\right)\right] } \\
& \geq {\left[*_{i=0}^{m-1} \mathfrak{m}_{\mathrm{h}}\left(c_{0}, c_{1}, \frac{t / 5^{i+1}}{\beta^{n+4 i+2} \prod_{j=0}^{i} b\left(c_{4 j}, c_{4 m+2}\right)}\right)\right]\left[*_{i=0}^{m-1} \mathfrak{m}_{\mathrm{h}}\left(c_{0}, c_{1}, \frac{t / 5^{i+1}}{\beta^{n+4 i+3} \prod_{j=0}^{i} b\left(c_{4 j}, c_{4 m+2}\right)}\right)\right] } \\
& * \mathfrak{m}_{\mathrm{h}}\left(c_{0}, c_{2}, \frac{t / 5^{m}}{\beta^{n+4 m} \prod_{j=0}^{m-1} b\left(c_{4 j}, c_{4 m+2}\right)}\right)
\end{aligned}
$$

Accordingly, Equations (13) and (14) can be altered as

$$
\begin{aligned}
& \mathfrak{m}_{\mathrm{h}}\left(c_{n}, c_{n+4 m+3}, t\right) \geq \mathfrak{m}_{\mathrm{h}}\left(c_{0}, c_{4 m+3}, \frac{t}{\beta^{n}}\right) \\
& \geq {\left[*_{i=0}^{m-1} \mathfrak{m}_{\mathrm{h}}\left(c_{0}, c_{1}, \frac{t / 5^{i+1}}{\beta^{n+4 i} \prod_{j=0}^{i} b\left(c_{4 j}, c_{4 m+3}\right)}\right)\right] *\left[*_{i=0}^{m-1} \mathfrak{m}_{\mathrm{h}}\left(c_{0}, c_{1}, \frac{t / 5^{i+1}}{\beta^{n+4 i+1} \prod_{j=0}^{i} b\left(c_{4 j}, c_{4 m+3}\right)}\right)\right] } \\
& * {\left[*_{i=0}^{m-1} \mathfrak{m}_{\mathrm{h}}\left(c_{0}, c_{1}, \frac{t / 5^{i+1}}{\beta^{n+4 i+2} \prod_{j=0}^{i} b\left(c_{4 j}, c_{4 m+3}\right)}\right)\right]\left[*_{i=0}^{m-1} \mathfrak{m}_{\mathrm{h}}\left(c_{0}, c_{1}, \frac{t / 5^{i+1}}{\beta^{n+4 i+3} \prod_{j=0}^{i} b\left(c_{4 j}, c_{4 m+3}\right)}\right)\right] } \\
& * \mathfrak{m}_{\mathrm{h}}\left(c_{0}, c_{3}, \frac{t / 5^{m}}{\beta^{n+4 m} \prod_{j=0}^{m-1} b\left(c_{4 j}, c_{4 m+3}\right)}\right)
\end{aligned}
$$

and

$$
\begin{aligned}
& \mathfrak{m}_{\mathrm{h}}\left(c_{n}, c_{n+4 m+4}, t\right) \geq \mathfrak{m}_{\mathrm{h}}\left(c_{0}, c_{4 m+4}, \frac{t}{\beta^{n}}\right) \\
& \geq {\left[*_{i=0}^{m-1} \mathfrak{m}_{\mathrm{h}}\left(c_{0}, c_{1}, \frac{t / 5^{i+1}}{\beta^{n+4 i} \prod_{j=0}^{i} b\left(c_{4 j}, c_{4 m+4}\right)}\right)\right] *\left[*_{i=0}^{m-1} \mathfrak{m}_{\mathrm{h}}\left(c_{0}, c_{1}, \frac{t / 5^{i+1}}{\beta^{n+4 i+1} \prod_{j=0}^{i} b\left(c_{4 j}, c_{4 m+4}\right)}\right)\right] } \\
& * {\left[*_{i=0}^{m-1} \mathfrak{m}_{\mathrm{h}}\left(c_{0}, c_{1}, \frac{t / 5^{i+1}}{\beta^{n+4 i+2} \prod_{j=0}^{i} b\left(c_{4 j}, c_{4 m+4}\right)}\right)\right]\left[*_{i=0}^{m-1} \mathfrak{m}_{\mathrm{h}}\left(c_{0}, c_{1}, \frac{t / 5^{i+1}}{\beta^{n+4 i+3} \prod_{j=0}^{i} b\left(c_{4 j}, c_{4 m+4}\right)}\right)\right] } \\
& * \mathfrak{m}_{\mathrm{h}}\left(c_{0}, c_{4}, \frac{t / 5^{m}}{\beta^{4 m} \prod_{j=0}^{m-1} b\left(c_{4 j}, c_{4 m+4}\right)}\right) .
\end{aligned}
$$

Therefore, for each $q$, along with the inequalities (15)-(18), we have

$$
\lim _{n \rightarrow \infty} \mathfrak{m}_{\mathrm{h}}\left(c_{n}, c_{n+q}, t\right)=1 * 1 * 1 * \ldots * 1=1,
$$


as $b\left(c_{n}, c_{n+q}\right) \beta<1$ for all $n, q \in \mathbb{N}$ and $\beta \in(0,1)$ i.e., $c_{n}$ is a Cauchy sequence in $X$. Seeing that $\left(X, \mathfrak{m}_{\mathrm{h}}, *\right)$ is complete, there exists some $c \in X$ such that $c_{n} \rightarrow c$ as $n \rightarrow \infty$. We assert that $c$ is the fixed point of $F$. Considering Equation (19) and condition $\left(\mathfrak{m}_{\mathrm{h}} 4\right)$, we get

$$
\begin{aligned}
\mathfrak{m}_{\mathrm{h}}(c, F c, t) \geq & \mathfrak{m}_{\mathrm{h}}\left(c, c_{n}, \frac{t / 5}{b(c, F c)}\right) * \mathfrak{m}_{\mathrm{h}}\left(F c_{n-1}, F c_{n}, \frac{t / 5}{b(c, F c)}\right) * \mathfrak{m}_{\mathrm{h}}\left(F c_{n}, F c_{n+1}, \frac{t / 5}{b(c, F c)}\right) \\
& * \mathfrak{m}_{\mathrm{h}}\left(F c_{n+1}, F c_{n+2}, \frac{t / 5}{b(c, F c)}\right) * \mathfrak{m}_{\mathrm{h}}\left(F c_{n+2}, F c, \frac{t / 5}{b(c, F c)}\right) \\
\geq & \mathfrak{m}_{\mathrm{h}}\left(c, c_{n}, \frac{t / 5}{b(c, F c)}\right) * \mathfrak{m}_{\mathrm{h}}\left(c_{n-1}, c_{n}, \frac{t / 5}{\beta b(c, F c)}\right) * \mathfrak{m}_{\mathrm{h}}\left(c_{n}, c_{n+1}, \frac{t / 5}{\beta b(c, F c)}\right) \\
& * \mathfrak{m}_{\mathrm{h}}\left(c_{n+1}, c_{n+2}, \frac{t / 5}{\beta b(c, F c)}\right) * \mathfrak{m}_{\mathrm{h}}\left(c_{n+2}, c, \frac{t / 5}{\beta b(c, F c)}\right) .
\end{aligned}
$$

Letting $n \rightarrow \infty$ in the above inequality, we get $F c=c$, i.e., $c$ is a fixed point of $F$. Through employing inequality (6), we can simply demonstrate that $c$ is the unique fixed point of $F$.

In [31], the authors have proposed the family of functions $\delta:[0, \infty) \rightarrow\left[0, \frac{1}{r}\right)$, which is represented by $\mathcal{P}_{r}$ as well as they meet the below criteria:

$$
\lim \sup _{n \rightarrow \infty} \delta\left(t_{n}\right)=\frac{1}{r} \text { implies } \lim _{n \rightarrow \infty} t_{n}=0,
$$

for any real number $r>1$.

In the context of fuzzy extended hexagonal $b$-metric spaces, we demonstrate a fixed point theorem as given below:

Theorem 2. Let $\left(X, \mathfrak{m}_{\mathrm{h}}, *\right)$ be a complete fuzzy extended hexagonal $b$-metric space and $b: X \times$ $X \rightarrow[1, \infty)$ such that

$$
\lim _{t \rightarrow \infty} \mathfrak{m}_{\mathrm{h}}(c, d, t)=1, \forall c, d \in X \text { and } t>0 .
$$

Let $F: X \rightarrow X$ be a mapping such that

$$
\mathfrak{m}_{\mathrm{h}}\left(F c, F d, \delta\left(\mathfrak{m}_{\mathrm{h}}(c, d, t)\right) t\right) \geq \mathfrak{m}_{\mathrm{h}}(c, d, t),
$$

for all $c, d \in X$ and for some $\delta \in \mathcal{P}_{r}$. Furthermore, if for $c_{0} \in X$ and $n, q \in \mathbb{N}$, it holds that $b\left(c_{n}, c_{n+q}\right)<r$ where $c_{n}=F^{n} c_{0}$, then $F$ has a unique fixed point.

Proof. Starting at the arbitrary point $c_{0} \in X$, construct the sequence $\left\{c_{n}\right\}$ in $X$ and continue the iterative procedure $c_{n+1}=F c_{n}$. For all $n, t>0$, we have

$$
\begin{aligned}
\mathfrak{m}_{\mathrm{h}}\left(c_{n}, c_{n+1}, t\right)= & \mathfrak{m}_{\mathrm{h}}\left(F c_{n-1}, F c_{n}, t\right) \\
\geq & \mathfrak{m}_{\mathrm{h}}\left(c_{n-1}, c_{n}, \frac{t}{\delta\left(\mathfrak{m}_{\mathrm{h}}\left(c_{n-1}, c_{n}, t\right)\right)}\right) \\
\geq & \mathfrak{m}_{\mathrm{h}}\left(c_{n-2}, c_{n-1}, \frac{t}{\delta\left(\mathfrak{m}_{\mathrm{h}}\left(c_{n-1}, c_{n}, t\right)\right) \delta\left(\mathfrak{m}_{\mathrm{h}}\left(c_{n-2}, c_{n-1}, t\right)\right)}\right) \\
& \vdots \\
\geq & \mathfrak{m}_{\mathrm{h}}\left(c_{0}, c_{1}, \frac{t}{\delta\left(\mathfrak{m}_{\mathrm{h}}\left(c_{n-1}, c_{n}, t\right)\right) \delta\left(\mathfrak{m}_{\mathrm{h}}\left(c_{n-2}, c_{n-1}, t\right)\right) \ldots \delta\left(\mathfrak{m}_{\mathrm{h}}\left(c_{0}, c_{1}, t\right)\right)}\right)
\end{aligned}
$$


and

$$
\begin{aligned}
\mathfrak{m}_{\mathrm{h}}\left(c_{n}, c_{n+2}, t\right)= & \mathfrak{m}_{\mathrm{h}}\left(F c_{n-1}, F c_{n+1}, t\right) \\
\geq & \mathfrak{m}_{\mathrm{h}}\left(c_{n-1}, c_{n+1}, \frac{t}{\delta\left(\mathfrak{m}_{\mathrm{h}}\left(c_{n-1}, c_{n+1}, t\right)\right)}\right) \\
\geq & \mathfrak{m}_{\mathrm{h}}\left(c_{n-2}, c_{n}, \frac{t}{\delta\left(\mathfrak{m}_{\mathrm{h}}\left(c_{n-1}, c_{n+1}, t\right)\right) \delta\left(\mathfrak{m}_{\mathrm{h}}\left(c_{n-2}, c_{n}, t\right)\right)}\right) \\
& \vdots \\
\geq & \mathfrak{m}_{\mathrm{h}}\left(c_{0}, c_{2}, \frac{t}{\delta\left(\mathfrak{m}_{\mathrm{h}}\left(c_{n-1}, c_{n+1}, t\right)\right) \delta\left(\mathfrak{m}_{\mathrm{h}}\left(c_{n-2}, c_{n}, t\right)\right) \ldots \delta\left(\mathfrak{m}_{\mathrm{h}}\left(c_{0}, c_{2}, t\right)\right)}\right) .
\end{aligned}
$$

By following the same technique, we get

$$
\begin{aligned}
& \mathfrak{m}_{\mathrm{h}}\left(c_{n}, c_{n+3}, t\right) \geq \mathfrak{m}_{\mathrm{h}}\left(c_{0}, c_{3}, \frac{t}{\delta\left(\mathfrak{m}_{\mathrm{h}}\left(c_{n-1}, c_{n+2}, t\right)\right) \delta\left(\mathfrak{m}_{\mathrm{h}}\left(c_{n-2}, c_{n+1}, t\right)\right) \ldots \delta\left(\mathfrak{m}_{\mathrm{h}}\left(c_{0}, c_{3}, t\right)\right)}\right) \\
& \mathfrak{m}_{\mathrm{h}}\left(c_{n}, c_{n+4}, t\right) \geq \mathfrak{m}_{\mathrm{h}}\left(c_{0}, c_{4}, \frac{t}{\delta\left(\mathfrak{m}_{\mathrm{h}}\left(c_{n-1}, c_{n+3}, t\right)\right) \delta\left(\mathfrak{m}_{\mathrm{h}}\left(c_{n-2,}, c_{n+2}, t\right)\right) \ldots \delta\left(\mathfrak{m}_{\mathrm{h}}\left(c_{0}, c_{4}, t\right)\right)}\right)
\end{aligned}
$$

To verify that the sequence $\left\{c_{n}\right\}$ is Cauchy, we must evaluate the inequalities below by applying the hexagonal inequality mentioned in $\left(\mathfrak{m}_{\mathrm{h}} 4\right)$. Consider

$$
\begin{aligned}
& \mathfrak{m}_{\mathrm{h}}\left(c_{n}, c_{n+4 m+1}, t\right) \geq \mathfrak{m}_{\mathrm{h}}\left(c_{n}, c_{n+1}, \frac{t / 5}{b\left(c_{n}, c_{n+4 m+1}\right)}\right) * \mathfrak{m}_{\mathrm{h}}\left(c_{n+1}, c_{n+2}, \frac{t / 5}{b\left(c_{n}, c_{n+4 m+1}\right)}\right) \\
& * \mathfrak{m}_{\mathrm{h}}\left(c_{n+2}, c_{n+3}, \frac{t / 5}{b\left(c_{n}, c_{n+4 m+1}\right)}\right) * \mathfrak{m}_{\mathrm{h}}\left(c_{n+3}, c_{n+4}, \frac{t / 5}{b\left(c_{n}, c_{n+4 m+1}\right)}\right) \\
& * \mathfrak{m}_{\mathrm{h}}\left(c_{n+4}, c_{n+5}, \frac{t / 5^{2}}{b\left(c_{n}, c_{n+4 m+1}\right) b\left(c_{n+4}, c_{n+4 m+1}\right)}\right) \\
& * \mathfrak{m}_{\mathrm{h}}\left(c_{n+5}, c_{n+6}, \frac{t / 5^{2}}{b\left(c_{n}, c_{n+4 m+1}\right) b\left(c_{n+4}, c_{n+4 m+1}\right)}\right) \\
& * \mathfrak{m}_{\mathrm{h}}\left(c_{n+4 m-4}, c_{n+4 m-3}, \frac{t / 5^{m}}{b\left(c_{n}, c_{n+4 m+1}\right) b\left(c_{n+4}, c_{n+4 m+1}\right) \ldots b\left(c_{n+4 m-4}, c_{n+4 m+1}\right)}\right) \\
& * \mathfrak{m}_{\mathrm{h}}\left(c_{n+4 m-3}, c_{n+4 m-2}, \frac{t / 5^{m}}{b\left(c_{n}, c_{n+4 m+1}\right) b\left(c_{n+4}, c_{n+4 m+1}\right) \ldots b\left(c_{n+4 m-4}, c_{n+4 m+1}\right)}\right) \\
& * \mathfrak{m}_{\mathrm{h}}\left(c_{n+4 m-2}, c_{n+4 m-1}, \frac{t / 5^{m}}{b\left(c_{n}, c_{n+4 m+1}\right) b\left(c_{n+4}, c_{n+4 m+1}\right) \ldots b\left(c_{n+4 m-4}, c_{n+4 m+1}\right)}\right) \\
& * \mathfrak{m}_{\mathrm{h}}\left(c_{n+4 m-1}, c_{n+4 m}, \frac{t / 5^{m}}{b\left(c_{n}, c_{n+4 m+1}\right) b\left(c_{n+4}, c_{n+4 m+1}\right) \ldots b\left(c_{n+4 m-4}, c_{n+4 m+1}\right)}\right) \\
& * \mathfrak{m}_{\mathrm{h}}\left(c_{n+4 m}, c_{n+4 m+1}, \frac{t / 5^{m}}{b\left(c_{n}, c_{n+4 m+1}\right) b\left(c_{n+4}, c_{n+4 m+1}\right) \ldots b\left(c_{n+4 m-4}, c_{n+4 m+1}\right)}\right) \\
& \geq \mathfrak{m}_{\mathrm{h}}\left(c_{0}, c_{1}, \frac{t / 5}{\delta\left(\mathfrak{m}_{\mathrm{h}}\left(c_{n-1}, c_{n}, t\right)\right) \delta\left(\mathfrak{m}_{\mathrm{h}}\left(c_{n-2}, c_{n-1}, t\right)\right) \ldots \delta\left(\mathfrak{m}_{\mathrm{h}}\left(c_{0}, c_{1}, t\right)\right) b\left(c_{n}, c_{n+4 m+1}\right)}\right) \\
& * \mathfrak{m}_{\mathrm{h}}\left(c_{0}, c_{1}, \frac{t / 5}{\delta\left(\mathfrak{m}_{\mathrm{h}}\left(c_{n}, c_{n+1}, t\right)\right) \delta\left(\mathfrak{m}_{\mathrm{h}}\left(c_{n-1}, c_{n}, t\right)\right) \ldots \delta\left(\mathfrak{m}_{\mathfrak{h}}\left(c_{0}, c_{1}, t\right)\right) b\left(c_{n}, c_{n+4 m+1}\right)}\right)
\end{aligned}
$$




$$
\begin{aligned}
& * \mathfrak{m}_{\mathrm{h}}\left(c_{0}, c_{1}, \frac{t / 5}{\delta\left(\mathfrak{m}_{\mathrm{h}}\left(c_{n+1}, c_{n+2}, t\right)\right) \delta\left(\mathfrak{m}_{\mathrm{h}}\left(c_{n}, c_{n+1}, t\right)\right) \ldots \delta\left(\mathfrak{m}_{\mathrm{h}}\left(c_{0}, c_{1}, t\right)\right) b\left(c_{n}, c_{n+4 m+1}\right)}\right) \\
& * \mathfrak{m}_{\mathrm{h}}\left(c_{0}, c_{1}, \frac{t / 5}{\delta\left(\mathfrak{m}_{\mathrm{h}}\left(c_{n+2}, c_{n+3}, t\right)\right) \delta\left(\mathfrak{m}_{\mathrm{h}}\left(c_{n+1}, c_{n+2}, t\right)\right) \ldots \delta\left(\mathfrak{m}_{\mathrm{h}}\left(c_{0}, c_{1}, t\right)\right) b\left(c_{n}, c_{n+4 m+1}\right)}\right) \\
& * \mathfrak{m}_{\mathrm{h}}\left(c_{0}, c_{1}, \frac{t / 5}{\delta\left(\mathfrak{m}_{\mathrm{h}}\left(c_{n+3}, c_{n+4}, t\right)\right) \delta\left(\mathfrak{m}_{\mathrm{h}}\left(c_{n+2}, c_{n+3}, t\right)\right) \ldots \delta\left(\mathfrak{m}_{\mathrm{h}}\left(c_{0}, c_{1}, t\right)\right) b\left(c_{n}, c_{n+4 m+1}\right)}\right) \\
& \text { : } \\
& * \mathfrak{m}_{\mathrm{h}}\left(c_{0}, c_{1}, \frac{t / 5^{m}}{\delta\left(\mathfrak{m}_{\mathrm{h}}\left(c_{n+4 m-2}, c_{n+4 m-1}, t\right)\right) \delta\left(\mathfrak{m}_{\mathrm{h}}\left(c_{n+4 m-3}, c_{n+4 m-2}, t\right)\right) \ldots \delta\left(\mathfrak{m}_{\mathrm{h}}\left(c_{0}, c_{1}, t\right)\right) \prod_{i=0}^{m-1} b\left(c_{n+4 i}, c_{n+4 m+1}\right)}\right) \\
& * \mathfrak{m}_{\mathrm{h}}\left(c_{0}, c_{1}, \frac{t / 5^{m}}{\delta\left(\mathfrak{m}_{\mathrm{h}}\left(c_{n+4 m-1}, c_{n+4 m}, t\right)\right) \delta\left(\mathfrak{m}_{\mathrm{h}}\left(c_{n+4 m-2}, c_{n+4 m-1}, t\right)\right) \ldots \delta\left(\mathfrak{m}_{\mathrm{h}}\left(c_{0}, c_{1}, t\right)\right) \prod_{i=0}^{m-1} b\left(c_{n+4 i}, c_{n+4 m+1}\right)}\right) \\
& \geq \mathfrak{m}_{\mathrm{h}}\left(c_{0}, c_{1}, \frac{t r^{n}}{5 b\left(c_{n}, c_{n+4 m+1}\right)}\right) * \mathfrak{m}_{\mathrm{h}}\left(c_{0}, c_{1}, \frac{t r^{n+1}}{5 b\left(c_{n}, c_{n+4 m+1}\right)}\right) * \mathfrak{m}_{\mathrm{h}}\left(c_{0}, c_{1}, \frac{t r^{n+2}}{5 b\left(c_{n}, c_{n+4 m+1}\right)}\right) \\
& * \cdots * \mathfrak{m}_{\mathrm{h}}\left(c_{0}, c_{1}, \frac{t r^{n+4 m-1}}{5^{m} \prod_{i=0}^{m-1} b\left(c_{n+4 i}, c_{n+4 m+1}\right)}\right) * \mathfrak{m}_{\mathrm{h}}\left(c_{0}, c_{1}, \frac{t r^{n+4 m}}{5^{m} \prod_{i=0}^{m-1} b\left(c_{n+4 i}, c_{n+4 m+1}\right)}\right) \text {. }
\end{aligned}
$$

As $b\left(c_{n}, c_{n+q}\right)<r, \forall n, q \in \mathbb{N}$, the above inequality yields

$$
\begin{aligned}
\mathfrak{m}_{\mathrm{h}}\left(c_{n}, c_{n+4 m+1}, t\right) \geq & \mathfrak{m}_{\mathrm{h}}\left(c_{0}, c_{1}, \frac{t r^{n-1}}{5}\right) * \mathfrak{m}_{\mathrm{h}}\left(c_{0}, c_{1}, \frac{t r^{n}}{5}\right) * \mathfrak{m}_{\mathrm{h}}\left(c_{0}, c_{1}, \frac{t r^{n+1}}{5}\right) * \ldots * \mathfrak{m}_{\mathrm{h}}\left(c_{0}, c_{1}, \frac{t r^{n+3 m-1}}{5^{m}}\right) \\
& * \mathfrak{m}_{\mathrm{h}}\left(c_{0}, c_{1}, \frac{t r^{n+3 m}}{5^{m}}\right) .
\end{aligned}
$$


Symmetry 2021, 13, 2032

13 of 22

Consider

$$
\begin{aligned}
& \mathfrak{m}_{\mathrm{h}}\left(c_{n}, c_{n+4 m+2}, t\right) \geq\left[\mathfrak{m}_{\mathrm{h}}\left(c_{n}, c_{n+2}, \frac{t / 5}{b\left(c_{n}, c_{n+4 m+2}\right)}\right)\right] *\left[*_{i=2}^{4} \mathfrak{m}_{\mathrm{h}}\left(c_{n+i}, c_{n+i+1}, \frac{t / 5}{b\left(c_{n}, c_{n+4 m+2}\right)}\right)\right] \\
& *\left[*_{i=5}^{8} \mathfrak{m}_{\mathrm{h}}\left(c_{n+i}, c_{n+i+1}, \frac{t / 5^{2}}{b\left(c_{n}, c_{n+4 m+2}\right) b\left(c_{n+5}, c_{n+4 m+2}\right)}\right)\right] \\
& *\left[*_{i=4 m-3}^{4 m+1} \mathfrak{m}_{\mathrm{h}}\left(c_{n+i}, c_{n+i+1}, \frac{t / 5^{m}}{b\left(c_{n}, c_{n+4 m+2}\right) b\left(c_{n+5}, c_{n+4 m+2}\right) \ldots b\left(c_{n+4 m-3}, c_{n+4 m+2}\right)}\right)\right] \\
& \geq \mathfrak{m}_{\mathrm{h}}\left(c_{0}, c_{2}, \frac{t}{\left(\begin{array}{c}
\left.\delta\left(\mathfrak{m}_{\mathrm{h}}\left(c_{n-1}, c_{n+1}, t\right)\right) \delta\left(\mathfrak{m}_{\mathrm{h}}\left(c_{n-2}, c_{n}, t\right)\right) \ldots\right) \\
\delta\left(\mathfrak{m}_{\mathrm{h}}\left(c_{0}, c_{2}, t\right)\right) b\left(c_{n}, c_{n+4 m+2}\right)
\end{array}\right)}\right) \\
& *\left[*_{i=2}^{4} \mathfrak{m}_{\mathrm{h}}\left(c_{0}, c_{1}, \frac{t}{\left(\begin{array}{c}
\delta\left(\mathfrak{m}_{\mathrm{h}}\left(c_{n+i-1}, c_{n+i}, t\right)\right) \delta\left(\mathfrak{m}_{\mathrm{h}}\left(c_{n+i-2}, c_{n+i-1}, t\right)\right) \cdots \\
\delta\left(\mathfrak{m}_{\mathrm{h}}\left(c_{0}, c_{1}, t\right)\right) b\left(c_{n}, c_{n+4 m+2}\right)
\end{array}\right)}\right)\right] \\
& \text { : } \\
& *\left[*_{i=4 m-3}^{4 m+1} \mathfrak{m}_{\mathrm{h}}\left(c_{0}, c_{1}, \frac{t / 5^{m}}{\left(\begin{array}{c}
\delta\left(\mathfrak{m}_{\mathrm{h}}\left(c_{n+i-1}, c_{n+i}, t\right)\right) \delta\left(\mathfrak{m}_{\mathrm{h}}\left(c_{n+i-2}, c_{n+i-1}, t\right)\right) \ldots \delta\left(\mathfrak{m}_{\mathrm{h}}\left(c_{0}, c_{1}, t\right)\right) \\
\times b\left(c_{n}, c_{n+4 m+2}\right) b\left(c_{n+5}, c_{n+4 m+2}\right) \ldots b\left(c_{n+4 m-3}, c_{n+4 m+2}\right)
\end{array}\right)}\right)\right] \\
& \geq \mathfrak{m}_{\mathrm{h}}\left(c_{0}, c_{2}, \frac{t r^{n}}{5 b\left(c_{n}, c_{n+4 m+2}\right)}\right) *\left[*_{i=2}^{4} \mathfrak{m}_{\mathrm{h}}\left(c_{0}, c_{1}, \frac{t r^{n+i}}{5 b\left(c_{n}, c_{n+4 m+2}\right)}\right)\right] \\
& \text { : } \\
& {\left[*_{i=4 m-3}^{4 m+1} \mathfrak{m}_{\mathrm{h}}\left(c_{0}, c_{1}, \frac{t r^{n+i}}{5^{m} b\left(c_{n}, c_{n+4 m+2}\right) b\left(c_{n+5}, c_{n+4 m+2}\right) \ldots b\left(c_{n+4 m-3}, c_{n+4 m+2}\right)}\right)\right] .}
\end{aligned}
$$

Thus

$$
\mathfrak{m}_{\mathrm{h}}\left(c_{n}, c_{n+4 m+2}, t\right) \geq \mathfrak{m}_{\mathrm{h}}\left(c_{0}, c_{2}, \frac{t r^{n-1}}{5}\right) *\left[*_{i=2}^{4} \mathfrak{m}_{\mathrm{h}}\left(c_{0}, c_{1}, \frac{t r^{n+i-1}}{5}\right)\right] * \ldots *\left[*_{i=4 m-3}^{4 m+1} \mathfrak{m}_{\mathrm{h}}\left(c_{0}, c_{1}, \frac{t r^{n-m+i}}{5^{m}}\right)\right] .
$$

Similarly, we get

$$
\begin{aligned}
\mathfrak{m}_{\mathrm{h}}\left(c_{n}, c_{n+4 m+3}, t\right) \geq & {\left[\mathfrak{m}_{\mathrm{h}}\left(c_{n}, c_{n+3}, \frac{t / 5}{b\left(c_{n}, c_{n+4 m+3}\right)}\right)\right] *\left[*_{i=3}^{5} \mathfrak{m}_{\mathrm{h}}\left(c_{n+i}, c_{n+i+1}, \frac{t / 5}{b\left(c_{n}, c_{n+4 m+3}\right)}\right)\right] } \\
& *\left[*_{i=6}^{9} \mathfrak{m}_{\mathrm{h}}\left(c_{n+i}, c_{n+i+1}, \frac{t / 5^{2}}{b\left(c_{n}, c_{n+4 m+3}\right) b\left(c_{n+6}, c_{n+4 m+3}\right)}\right)\right] \\
& \vdots \\
& *\left[*_{i=4 m-2}^{4 m+2} \mathfrak{m}_{\mathrm{h}}\left(c_{n+i}, c_{n+i+1}, \frac{t / 5^{m}}{b\left(c_{n}, c_{n+4 m+3}\right) b\left(c_{n+6}, c_{n+4 m+3}\right) \ldots b\left(c_{n+4 m-2}, c_{n+4 m+3}\right)}\right)\right] \\
\geq & \mathfrak{m}_{\mathrm{h}}\left(c_{0}, c_{3}, \frac{t r^{n-1}}{5}\right) *\left[*_{i=3}^{5} \mathfrak{m}_{\mathrm{h}}\left(c_{0}, c_{1}, \frac{t r^{n+i-1}}{5}\right)\right] *\left[*_{i=6}^{9} \mathfrak{m}_{\mathrm{h}}\left(c_{0}, c_{1}, \frac{t r^{n+i-1}}{5}\right)\right] \\
& * \ldots *\left[*_{i=4 m-2}^{4 m+2} \mathfrak{m}_{\mathrm{h}}\left(c_{0}, c_{1}, \frac{t r^{n-m+i}}{5^{m}}\right)\right]
\end{aligned}
$$

and

$$
\mathfrak{m}_{\mathrm{h}}\left(c_{n}, c_{n+4 m+4}, t\right) \geq\left[\mathfrak{m}_{\mathrm{h}}\left(c_{n}, c_{n+4}, \frac{t / 5}{b\left(c_{n}, c_{n+4 m+4}\right)}\right)\right] *\left[*_{i=4}^{6} \mathfrak{m}_{\mathrm{h}}\left(c_{n+i}, c_{n+i+1}, \frac{t / 5}{b\left(c_{n}, c_{n+4 m+4}\right)}\right)\right]
$$




$$
\begin{aligned}
* & {\left[*_{i=7}^{10} \mathfrak{m}_{\mathrm{h}}\left(c_{n+i}, c_{n+i+1}, \frac{t / 5^{2}}{b\left(c_{n}, c_{n+4 m+4}\right) b\left(c_{n+7}, c_{n+4 m+4}\right)}\right)\right] } \\
& \vdots \\
* & {\left[*_{i=4 m-1}^{4 m+3} \mathfrak{m}_{\mathrm{h}}\left(c_{n+i}, c_{n+i+1}, \frac{t / 5^{m}}{b\left(c_{n}, c_{n+4 m+4}\right) b\left(c_{n+7}, c_{n+4 m+4}\right) \ldots b\left(c_{n+4 m-1}, c_{n+4 m+4}\right)}\right)\right] } \\
\geq & \mathfrak{m}_{\mathrm{h}}\left(c_{0}, c_{4}, \frac{t r^{n-1}}{5}\right) *\left[*_{i=4}^{6} \mathfrak{m}_{\mathrm{h}}\left(c_{0}, c_{1}, \frac{t r^{n+i-1}}{5}\right)\right] *\left[*_{i=7}^{10} \mathfrak{m}_{\mathrm{h}}\left(c_{0}, c_{1}, \frac{t r^{n+i-1}}{5}\right)\right] \\
& * \ldots *\left[*_{i=4 m-1}^{4 m+3} \mathfrak{m}_{\mathrm{h}}\left(c_{0}, c_{1}, \frac{t r^{n-m+i}}{5^{m}}\right)\right] .
\end{aligned}
$$

Each $q \in \mathbb{N}$, through the inequalities (26)-(29) together with (20), implies that

$$
\lim _{n \rightarrow \infty} \mathfrak{m}_{\mathrm{h}}\left(c_{n}, c_{n+q}, t\right)=1 * 1 * 1 * \ldots * 1=1,
$$

as $b\left(c_{n}, c_{n+q}\right)<r$ for all $n, q \in \mathbb{N}$. Henceforth, $\left\{c_{n}\right\}$ is a Cauchy sequence in $X$. From the completeness of $\left(X, \mathfrak{m}_{\mathrm{h}}, *\right)$, there exists $c \in X$ such that

$$
\lim _{n \rightarrow \infty} \mathfrak{m}_{\mathrm{h}}\left(c_{n}, c, t\right)=1
$$

for all $t>0$. Finally, we demonstrate that $c$ is a fixed point of $F$. For any $t>0$ and from the condition $\left(\mathfrak{m}_{\mathrm{h}} 4\right)$, we have

$$
\begin{aligned}
\mathfrak{m}_{\mathrm{h}}(c, F c, t) \geq & \mathfrak{m}_{\mathrm{h}}\left(c, F c_{n-1}, \frac{t / 5}{b(c, F c)}\right) * \mathfrak{m}_{\mathrm{h}}\left(F c_{n-1}, F c_{n}, \frac{t / 5}{b(c, F c)}\right) * \mathfrak{m}_{\mathrm{h}}\left(F c_{n}, F c_{n+1}, \frac{t / 5}{b(c, F c)}\right) \\
& * \mathfrak{m}_{\mathrm{h}}\left(F c_{n+1}, F c_{n+2}, \frac{t / 5}{b(c, F c)}\right) * \mathfrak{m}_{\mathrm{h}}\left(F c_{n+2}, F c, \frac{t / 5}{b(c, F c)}\right) \\
\geq & \mathfrak{m}_{\mathrm{h}}\left(c, c_{n}, \frac{t / 5}{b(c, F c)}\right) * \mathfrak{m}_{\mathrm{h}}\left(c_{n-1}, c_{n}, \frac{t / 5}{\delta\left(\mathfrak{m}_{\mathrm{h}}\left(c_{n-1}, c_{n}, t\right)\right) b(c, F c)}\right) \\
* & \mathfrak{m}_{\mathrm{h}}\left(c_{n}, c_{n+1}, \frac{t / 5}{\delta\left(\mathfrak{m}_{\mathrm{h}}\left(c_{n}, c_{n+1}, t\right)\right) b(c, F c)}\right) * \mathfrak{m}_{\mathrm{h}}\left(c_{n+1}, c_{n+2}, \frac{t / 5}{\delta\left(\mathfrak{m}_{\mathrm{h}}\left(c_{n+1}, c_{n+2}, t\right)\right) b(c, F c)}\right) \\
* & \mathfrak{m}_{\mathrm{h}}\left(c_{n+2}, c, \frac{t / 5}{\delta\left(\mathfrak{m}_{\mathrm{h}}\left(c_{n+2, c, t)) b(c, F c)}\right) .\right.}\right.
\end{aligned}
$$

Letting $n \rightarrow \infty$ in the preceding inequality and employing (30) and (31), we get $\mathfrak{m}_{\mathrm{h}}(c, F c, t)=1$ for all $t>0$ i.e., $c=F c$. By considering the inequality (21), we obtain that $c$ is the unique fixed point of $F$.

In [32], Mihet described the following class of functions, namely fuzzy $\Psi$-contractive mappings.

Definition 10. Let $\Psi$ be the class of all mappings $\psi:[0,1] \rightarrow[0,1]$ such that $\psi$ is continuous, nondecreasing, and $\psi(a)>a$ for all $a \in(0,1)$. If $\psi \in \Psi$, then $\psi(1)=1$ and $\lim _{n \rightarrow \infty} \psi^{n}(a)=1$ for all $a \in(0,1)$.

Considering the above-mentioned definition, we establish the following significant result.

Theorem 3. Let $\left(X, \mathfrak{m}_{\mathrm{h}}, *\right)$ be a complete fuzzy extended hexagonal b-metric space. Let $F: X \rightarrow X$ be a mapping such that

$$
\mathfrak{m}_{\mathrm{h}}(c, d, t)>0 \Rightarrow \mathfrak{m}_{\mathrm{h}}(F c, F d, t) \geq \psi\left(\mathfrak{m}_{\mathrm{h}}(c, d, t)\right), \forall c, d \in X \text { and } t>0
$$


Then, F has a unique fixed point in X.

Proof. Let $c_{0} \in X$ and define a sequence $\left\{c_{n}\right\}$ by

$$
c_{n}=F c_{n-1} \text { for all } n \in \mathbb{N} \text {. }
$$

Without losing generality, assume that $c_{n} \neq c_{n+1}, \forall n \geq 0$. Consecutively, applying inequality (32), we deduce

$$
\begin{aligned}
\mathfrak{m}_{\mathrm{h}}\left(c_{n}, c_{n+1}, t\right) & =\mathfrak{m}_{\mathrm{h}}\left(F c_{n-1}, F c_{n}, t\right) \\
& \geq \psi\left(\mathfrak{m}_{\mathrm{h}}\left(c_{n-1}, c_{n}, t\right)\right) \\
& \vdots \\
& \geq \psi^{n}\left(\mathfrak{m}_{\mathrm{h}}\left(c_{0}, c_{1}, t\right)\right) .
\end{aligned}
$$

In a similar way, we acquire

$$
\begin{aligned}
& \mathfrak{m}_{\mathrm{h}}\left(c_{n}, c_{n+2}, t\right) \geq \psi^{n}\left(\mathfrak{m}_{\mathrm{h}}\left(c_{0}, c_{2}, t\right)\right), \\
& \mathfrak{m}_{\mathrm{h}}\left(c_{n}, c_{n+3}, t\right) \geq \psi^{n}\left(\mathfrak{m}_{\mathrm{h}}\left(c_{0}, c_{3}, t\right)\right),
\end{aligned}
$$

and

$$
\mathfrak{m}_{\mathrm{h}}\left(c_{n}, c_{n+4}, t\right) \geq \psi^{n}\left(\mathfrak{m}_{\mathrm{h}}\left(c_{0}, c_{4}, t\right)\right) .
$$

By applying the condition $\left(\mathfrak{m}_{\mathrm{h}} 4\right)$, we obtain

$$
\mathfrak{m}_{\mathrm{h}}\left(c_{n}, c_{n+4 m+1}, t\right) \geq \mathfrak{m}_{\mathrm{h}}\left(c_{n}, c_{n+1}, \frac{t / 5}{b\left(c_{n}, c_{n+4 m+1}\right)}\right) * \mathfrak{m}_{\mathrm{h}}\left(c_{n+1}, c_{n+2}, \frac{t / 5}{b\left(c_{n}, c_{n+4 m+1}\right)}\right)
$$




$$
\begin{aligned}
& * \mathfrak{m}_{\mathrm{h}}\left(c_{n+2}, c_{n+3}, \frac{t / 5}{b\left(c_{n}, c_{n+4 m+1}\right)}\right) * \mathfrak{m}_{\mathrm{h}}\left(c_{n+3}, c_{n+4}, \frac{t / 5}{b\left(c_{n}, c_{n+4 m+1}\right)}\right) * \mathfrak{m}_{\mathrm{h}}\left(c_{n+4}, c_{n+4 m+1}, \frac{t / 5}{b\left(c_{n}, c_{n+4 m+1}\right)}\right) \\
& \geq \mathfrak{m}_{\mathrm{h}}\left(c_{n}, c_{n+1}, \frac{t / 5}{b\left(c_{n}, c_{n+4 m+1}\right)}\right) * \mathfrak{m}_{\mathrm{h}}\left(c_{n+1}, c_{n+2}, \frac{t / 5}{b\left(c_{n}, c_{n+4 m+1}\right)}\right) \\
& * \mathfrak{m}_{\mathrm{h}}\left(c_{n+2}, c_{n+3}, \frac{t / 5}{b\left(c_{n}, c_{n+4 m+1}\right)}\right) * \mathfrak{m}_{\mathrm{h}}\left(c_{n+3}, c_{n+4}, \frac{t / 5}{b\left(c_{n}, c_{n+4 m+1}\right)}\right) \\
& * \mathfrak{m}_{\mathrm{h}}\left(c_{n+4}, c_{n+5}, \frac{t / 5^{2}}{b\left(c_{n}, c_{n+4 m+1}\right) b\left(c_{n+4}, c_{n+4 m+1}\right)}\right) \\
& * \mathfrak{m}_{\mathrm{h}}\left(c_{n+5}, c_{n+6}, \frac{t / 5^{2}}{b\left(c_{n}, c_{n+4 m+1}\right) b\left(c_{n+4}, c_{n+4 m+1}\right)}\right) \\
& * \mathfrak{m}_{\mathrm{h}}\left(c_{n+6}, c_{n+7}, \frac{t / 5^{2}}{b\left(c_{n}, c_{n+4 m+1}\right) b\left(c_{n+4}, c_{n+4 m+1}\right)}\right) \\
& * \mathfrak{m}_{\mathrm{h}}\left(c_{n+7}, c_{n+8}, \frac{t / 5^{2}}{b\left(c_{n}, c_{n+4 m+1}\right) b\left(c_{n+4}, c_{n+4 m+1}\right)}\right) \\
& \text { : } \\
& * \mathfrak{m}_{\mathrm{h}}\left(c_{n+4 m-4}, c_{n+4 m-3}, \frac{t / 5^{m}}{b\left(c_{n}, c_{n+4 m+1}\right) b\left(c_{n+4}, c_{n+4 m+1}\right) \ldots b\left(c_{n+4 m-4}, c_{n+4 m+1}\right)}\right) \\
& * \mathfrak{m}_{\mathrm{h}}\left(c_{n+4 m-3}, c_{n+4 m-2}, \frac{t / 5^{m}}{b\left(c_{n}, c_{n+4 m+1}\right) b\left(c_{n+4}, c_{n+4 m+1}\right) \ldots b\left(c_{n+4 m-4}, c_{n+4 m+1}\right)}\right) \\
& * \mathfrak{m}_{\mathrm{h}}\left(c_{n+4 m-2}, c_{n+4 m-1}, \frac{t / 5^{m}}{b\left(c_{n}, c_{n+4 m+1}\right) b\left(c_{n+4}, c_{n+4 m+1}\right) \ldots b\left(c_{n+4 m-4}, c_{n+4 m+1}\right)}\right) \\
& * \mathfrak{m}_{\mathrm{h}}\left(c_{n+4 m-1}, c_{n+4 m}, \frac{t / 5^{m}}{b\left(c_{n}, c_{n+4 m+1}\right) b\left(c_{n+4}, c_{n+4 m+1}\right) \ldots b\left(c_{n+4 m-4}, c_{n+4 m+1}\right)}\right) \\
& * \mathfrak{m}_{\mathrm{h}}\left(c_{n+4 m}, c_{n+4 m+1}, \frac{t / 5^{m}}{b\left(c_{n}, c_{n+4 m+1}\right) b\left(c_{n+4}, c_{n+4 m+1}\right) \ldots b\left(c_{n+4 m-4}, c_{n+4 m+1}\right)}\right) \\
& \geq\left[*_{i=0}^{m-1} \psi^{n+4 i}\left(\mathfrak{m}_{\mathrm{h}}\left(c_{0}, c_{1}, \frac{t / 5^{i+1}}{\prod_{j=0}^{i} b\left(c_{n+4 j}, c_{n+4 m+1}\right)}\right)\right)\right] *\left[*_{i=0}^{m-1} \psi^{n+4 i+1}\left(\mathfrak{m}_{\mathrm{h}}\left(c_{0}, c_{1}, \frac{t / 5^{i+1}}{\prod_{j=0}^{i} b\left(c_{n+4 j}, c_{n+4 m+1}\right)}\right)\right)\right] \\
& *\left[*_{i=0}^{m-1} \psi^{n+4 i+2}\left(\mathfrak{m}_{\mathrm{h}}\left(c_{0}, c_{1}, \frac{t / 5^{i+1}}{\prod_{j=0}^{i} b\left(c_{n+4 j}, c_{n+4 m+1}\right)}\right)\right)\right] *\left[*_{i=0}^{m-1} \psi^{n+4 i+3}\left(\mathfrak{m}_{\mathrm{h}}\left(c_{0}, c_{1}, \frac{t / 5^{i+1}}{\prod_{j=0}^{i} b\left(c_{n+4 j}, c_{n+4 m+1}\right)}\right)\right)\right] \\
& * \psi^{n+4 m}\left(\mathfrak{m}_{\mathrm{h}}\left(c_{0}, c_{1}, \frac{t / 5^{m}}{\prod_{j=0}^{m-1} b\left(c_{n+4 j}, c_{n+4 m+1}\right)}\right)\right) .
\end{aligned}
$$

Similarly, $\mathfrak{m}_{\mathrm{h}}\left(c_{n}, c_{n+4 m+2}, t\right), \mathfrak{m}_{\mathrm{h}}\left(c_{n}, c_{n+4 m+3}, t\right)$ and $\mathfrak{m}_{\mathrm{h}}\left(c_{n}, c_{n+4 m+4}, t\right)$ can be derived as

$$
\begin{aligned}
\mathfrak{m}_{\mathrm{h}}\left(c_{n}, c_{n+4 m+2}, t\right) \geq & \psi^{n}\left(\mathfrak{m}_{\mathrm{h}}\left(c_{0}, c_{2}, \frac{t / 5}{b\left(c_{n}, c_{n+4 m+2}\right)}\right)\right) \\
* & {\left[*_{i=2}^{4} \psi^{n+i}\left(\mathfrak{m}_{\mathrm{h}}\left(c_{0}, c_{1}, \frac{t / 5^{2}}{b\left(c_{n}, c_{n+4 m+2}\right) b\left(c_{n+5}, c_{n+4 m+2}\right)}\right)\right)\right] } \\
& *\left[*_{i=5}^{8} \psi^{n+i}\left(\mathfrak{m}_{\mathrm{h}}\left(c_{0}, c_{1}, \frac{t / 5^{3}}{b\left(c_{n}, c_{n+4 m+2}\right) b\left(c_{n+5}, c_{n+4 m+2}\right) b\left(c_{n+9}, c_{n+4 m+2}\right)}\right)\right)\right] \\
& \vdots \\
& *\left[*_{i=4 m-3}^{4 m+1} \psi^{n+i}\left(\mathfrak{m}_{\mathrm{h}}\left(c_{0}, c_{1}, \frac{t / 5^{m}}{b\left(c_{n}, c_{n+4 m+2}\right) b\left(c_{n+5}, c_{n+4 m+2}\right) \ldots b\left(c_{n+4 m-3}, c_{n+4 m+2}\right)}\right)\right)\right],
\end{aligned}
$$




$$
\begin{aligned}
\mathfrak{m}_{\mathrm{h}}\left(c_{n}, c_{n+4 m+3}, t\right) \geq & \psi^{n}\left(\mathfrak{m}_{\mathrm{h}}\left(c_{0}, c_{3}, \frac{t / 5}{b\left(c_{n}, c_{n+4 m+3}\right)}\right)\right) \\
* & {\left[*_{i=3}^{5} \psi^{n+i}\left(\mathfrak{m}_{\mathrm{h}}\left(c_{0}, c_{1}, \frac{t / 5^{2}}{b\left(c_{n}, c_{n+4 m+3}\right) b\left(c_{n+6}, c_{n+4 m+3}\right)}\right)\right)\right] } \\
* & {\left[*_{i=6}^{9} \psi^{n+i}\left(\mathfrak{m}_{\mathrm{h}}\left(c_{0}, c_{1}, \frac{t / 5^{3}}{b\left(c_{n}, c_{n+4 m+3}\right) b\left(c_{n+6}, c_{n+4 m+3}\right) b\left(c_{n+10}, c_{n+4 m+3}\right)}\right)\right)\right] } \\
& \vdots \\
& *\left[*_{i=4 m-2}^{4 m+2} \psi^{n+i}\left(\mathfrak{m}_{\mathrm{h}}\left(c_{0}, c_{1}, \frac{t / 5^{m}}{b\left(c_{n}, c_{n+4 m+3}\right) b\left(c_{n+6}, c_{n+4 m+3}\right) \ldots b\left(c_{n+4 m-2}, c_{n+4 m+3}\right)}\right)\right)\right],
\end{aligned}
$$

and

$$
\begin{aligned}
\mathfrak{m}_{\mathrm{h}}\left(c_{n}, c_{n+4 m+4}, t\right) \geq & \psi^{n}\left(\mathfrak{m}_{\mathrm{h}}\left(c_{0}, c_{4}, \frac{t / 5}{b\left(c_{n}, c_{n+4 m+4}\right)}\right)\right) \\
& *\left[*_{i=4}^{6} \psi^{n+i}\left(\mathfrak{m}_{\mathrm{h}}\left(c_{0}, c_{1}, \frac{t / 5^{2}}{b\left(c_{n}, c_{n+4 m+4}\right) b\left(c_{n+7}, c_{n+4 m+4}\right)}\right)\right)\right] \\
& *\left[*_{i=7}^{10} \psi^{n+i}\left(\mathfrak{m}_{\mathrm{h}}\left(c_{0}, c_{1}, \frac{t / 5^{3}}{b\left(c_{n}, c_{n+4 m+4}\right) b\left(c_{n+7}, c_{n+4 m+4}\right) b\left(c_{n+11}, c_{n+4 m+4}\right)}\right)\right)\right] \\
& \vdots \\
& *\left[*_{i=4 m-1}^{4 m+3} \psi^{n+i}\left(\mathfrak{m}_{\mathrm{h}}\left(c_{0}, c_{1}, \frac{t / 5^{m}}{b\left(c_{n}, c_{n+4 m+4}\right) b\left(c_{n+7}, c_{n+4 m+4}\right) \ldots b\left(c_{n+4 m-1}, c_{n+4 m+4}\right)}\right)\right)\right] .
\end{aligned}
$$

Therefore, taking the limit $n \rightarrow \infty$ in each case and utilizing the definition of $\Psi$, we conclude that

$$
\lim _{n \rightarrow \infty} \mathfrak{m}_{\mathrm{h}}\left(c_{n}, c_{n+q}, t\right)=1 * 1 * 1 * \ldots * 1=1, \forall t>0 \text { and } n, q \in \mathbb{N}
$$

Consequently, $\left\{c_{n}\right\}$ is a Cauchy sequence in $X$. Based on the completeness of $\left(X, \mathfrak{m}_{\mathrm{h}}, *\right)$, there exists $c \in X$ such that

$$
\lim _{n \rightarrow \infty} \mathfrak{m}_{\mathrm{h}}\left(c_{n}, c, t\right)=1,
$$

for all $t>0$. Eventually, we show that $c$ is a fixed point of $F$. For any $t>0$ and from the condition $\left(\mathfrak{m}_{\mathrm{h}} 4\right)$, we find that

$$
\begin{aligned}
\mathfrak{m}_{\mathrm{h}}(c, F c, t) \geq & \mathfrak{m}_{\mathrm{h}}\left(c, c_{n}, \frac{t / 5}{b(c, F c)}\right) * \mathfrak{m}_{\mathrm{h}}\left(c_{n}, c_{n+1}, \frac{t / 5}{b(c, F c)}\right) * \mathfrak{m}_{\mathrm{h}}\left(c_{n+1}, c_{n+2}, \frac{t / 5}{b(c, F c)}\right) \\
& * \mathfrak{m}_{\mathrm{h}}\left(c_{n+2}, c_{n+3}, \frac{t / 5}{b(c, F c)}\right) * \mathfrak{m}_{\mathrm{h}}\left(c_{n+3}, F c, \frac{t / 5}{b(c, F c)}\right) \\
= & \mathfrak{m}_{\mathrm{h}}\left(c, c_{n}, \frac{t / 5}{b(c, F c)}\right) * \mathfrak{m}_{\mathrm{h}}\left(c_{n}, c_{n+1}, \frac{t / 5}{b(c, F c)}\right) * \mathfrak{m}_{\mathrm{h}}\left(c_{n+1}, c_{n+2}, \frac{t / 5}{b(c, F c)}\right) \\
& * \mathfrak{m}_{\mathrm{h}}\left(c_{n+2}, c_{n+3}, \frac{t / 5}{b(c, F c)}\right) * \mathfrak{m}_{\mathrm{h}}\left(F c_{n+2}, F c, \frac{t / 5}{b(c, F c)}\right), \\
\geq & \mathfrak{m}_{\mathrm{h}}\left(c, c_{n}, \frac{t / 5}{b(c, F c)}\right) * \mathfrak{m}_{\mathrm{h}}\left(c_{n}, c_{n+1}, \frac{t / 5}{b(c, F c)}\right) * \mathfrak{m}_{\mathrm{h}}\left(c_{n+1}, c_{n+2}, \frac{t / 5}{b(c, F c)}\right) \\
& * \mathfrak{m}_{\mathrm{h}}\left(c_{n+2}, c_{n+3}, \frac{t / 5}{b(c, F c)}\right) * \psi\left(\mathfrak{m}_{\mathrm{h}}\left(F c_{n+2}, F c, \frac{t / 5}{b(c, F c)}\right)\right) .
\end{aligned}
$$


Letting $n \rightarrow \infty$ in the above inequality and utilizing (34) and (35), we get $\mathfrak{m}_{\mathrm{h}}(c, F c, t)=1$ for all $t>0$ i.e., $c=F c$. By employing inequality (32), we can simply confirm that $c$ is the unique fixed point of $F$.

Example 2. Let $X=[0,2]$. Define $\mathfrak{m}_{\mathrm{h}}: X \times X \times[0, \infty) \rightarrow[0,1]$ as

$$
\mathfrak{m}_{\mathrm{h}}(\mu, v, t)=e^{-\frac{|\mu-v|^{6}}{t}}
$$

with the continuous $t$-norm ${ }^{*}$ such that $c_{1} * c_{2}=c_{1} \cdot c_{2}$. Evidently, $\left(X, \mathfrak{m}_{\mathrm{h}}, *\right)$ is a complete fuzzy extended hexagonal $b$-metric space with the control function $b(\mu, v)=1+|\mu-v|^{5}$. Define $F: X \rightarrow X$ by $F \mu=\frac{\mu}{2}$. Consider

$$
\begin{aligned}
\mathfrak{m}_{\mathrm{h}}(F \mu, F v, t)=e^{-\frac{|F \mu-F \nu|^{6}}{t}}=e^{-\frac{\left|\frac{\mu}{2}-\frac{\nu}{2}\right|^{6}}{t}}=e^{-\frac{1}{2^{6}} \frac{|\mu-\nu|^{6}}{t}} & \geq e^{-\frac{1}{2} \frac{|\mu-\nu|^{6}}{t}} \\
& =\left(e^{-\frac{\mid \mu-\nu 6^{6}}{t}}\right)^{\frac{1}{2}} \\
& =\psi\left(\mathfrak{m}_{\mathrm{h}}(\mu, v, t)\right),
\end{aligned}
$$

where $\psi:[0,1] \rightarrow[0,1]$ is specified by $\psi(\kappa)=\sqrt{\kappa}$ for all $\kappa \in[0,1]$. Hence, by Theorem 3, F has a unique fixed point in $\mathrm{X}$ which is $\mu=0$.

\section{Solution of Nonlinear Fractional Differential Equations: A Fixed Point Technique}

The important objective in this section is to apply Theorem 1 to study the existence and uniqueness of solutions to a nonlinear fractional differential equation (NFDE)

$$
D_{0+}^{\mathfrak{p}} y(t)=g(t, y(t)), 0<t<1
$$

with the boundary conditions $y(0)+y^{\prime}(0)=0, y(1)+y^{\prime}(1)=0$, where $1<\mathfrak{p} \leq 2$ is a real number, $D_{0+}^{\mathfrak{p}}$ is the Caputo fractional derivative and $g:[0,1] \times[0, \infty) \rightarrow[0, \infty)$ is a continuous function. Let $X=C([0,1], \mathbb{R})$ denote the space of all continuous functions defined on $[0,1]$ equipped with the product $t$-norm, i.e., $c * d=c . d$ for all $c, d \in[0,1]$ and specify the complete fuzzy extended hexagonal $b$-metric on $X$ as follows:

$$
\mathfrak{m}_{\mathrm{h}}(y, w, t)=\frac{\alpha t}{\alpha t+\gamma \sup _{\mathfrak{t} \in[0,1]}|y(\mathfrak{t})-w(\mathfrak{t})|^{6}}
$$

for all $t>0$ and $y, w \in X$ with the control function $b(y, w)=1+\sup _{\mathfrak{t} \in[0,1]}|y(\mathfrak{t})-w(\mathfrak{t})|^{5}$. Notice that $y \in X$ solves (36) whenever $y \in X$ solves the below integral equation:

$$
\begin{aligned}
y(t)= & \frac{1}{\Gamma(\mathfrak{p})} \int_{0}^{1}(1-s)^{\mathfrak{p}-1}(1-t) g(s, y(s)) d s+\frac{1}{\Gamma(\mathfrak{p}-1)} \int_{0}^{1}(1-s)^{\mathfrak{p}-2}(1-t) g(s, y(s)) d s \\
& +\frac{1}{\Gamma(\mathfrak{p})} \int_{0}^{t}(t-s)^{\mathfrak{p}-1} g(s, y(s)) d s .
\end{aligned}
$$

Regarding a more detailed description of the problem's context, the readers can adhere to the research [33]. The existence of a solution to the nonlinear fractional differential Equation (36) is demonstrated by the following theorem. 
Theorem 4. The integral operator $F: X \rightarrow X$ is given by

$$
\begin{aligned}
F y(t)= & \frac{1}{\Gamma(\mathfrak{p})} \int_{0}^{1}(1-s)^{\mathfrak{p}-1}(1-t) g(s, y(s)) d s+\frac{1}{\Gamma(\mathfrak{p}-1)} \int_{0}^{1}(1-s)^{\mathfrak{p}-2}(1-t) g(s, y(s)) d s \\
& +\frac{1}{\Gamma(\mathfrak{p})} \int_{0}^{t}(t-s)^{\mathfrak{p}-1} g(s, y(s)) d s,
\end{aligned}
$$

where $g:[0,1] \times[0, \infty) \rightarrow[0, \infty)$ fulfills the following criteria:

- $|g(s, y(s))-g(s, w(s))| \leq \frac{1}{4}|y(s)-w(s)|, \forall y, w \in X$;

- $\sup _{\mathfrak{t} \in(0,1)} \frac{1}{4096}\left[\frac{1-\mathfrak{t}}{\Gamma(\mathfrak{p}+1)}+\frac{1-\mathfrak{t}}{\Gamma(\mathfrak{p})}+\frac{\mathfrak{t}^{\mathfrak{p}}}{\Gamma(\mathfrak{p}+1)}\right]^{6}=\beta<1$.

Then, NFDE (36) has a unique solution in X:

\section{Proof.}

$$
\begin{aligned}
& |F y(t)-F w(t)|^{6}=\mid \frac{1-t}{\Gamma(\mathfrak{p})} \int_{0}^{1}(1-s)^{\mathfrak{p}-1}[g(s, y(s))-g(s, w(s))] d s \\
& +\frac{1-t}{\Gamma(\mathfrak{p}-1)} \int_{0}^{1}(1-s)^{\mathfrak{p}-2}[g(s, y(s))-g(s, w(s))] d s \\
& +\left.\frac{1}{\Gamma(\mathfrak{p})} \int_{0}^{t}(t-s)^{\mathfrak{p}-1}[g(s, y(s))-g(s, w(s))] d s\right|^{6} \\
& \leq\left(\frac{1-t}{\Gamma(\mathfrak{p})} \int_{0}^{1}(1-s)^{\mathfrak{p}-1}|g(s, y(s))-g(s, w(s))| d s\right. \\
& +\frac{1-t}{\Gamma(\mathfrak{p}-1)} \int_{0}^{1}(1-s)^{\mathfrak{p}-2}|g(s, y(s))-g(s, w(s))| d s \\
& \left.+\frac{1}{\Gamma(\mathfrak{p})} \int_{0}^{t}(t-s)^{\mathfrak{p}-1}|g(s, y(s))-g(s, w(s))| d s\right)^{6} \\
& \leq\left(\frac{1-t}{\Gamma(\mathfrak{p})} \int_{0}^{1}(1-s)^{\mathfrak{p}-1} \frac{|y(s)-w(s)|}{4} d s\right. \\
& +\frac{1-t}{\Gamma(\mathfrak{p}-1)} \int_{0}^{1}(1-s)^{\mathfrak{p}-2} \frac{|y(s)-w(s)|}{4} d s \\
& \left.+\frac{1}{\Gamma(\mathfrak{p})} \int_{0}^{t}(t-s)^{\mathfrak{p}-1} \frac{|y(s)-w(s)|}{4} d s\right)^{6} \\
& \leq \frac{1}{4^{6}} \sup _{\mathfrak{t} \in[0,1]}|y(\mathfrak{t})-w(\mathfrak{t})|^{6}\left(\frac{1-t}{\Gamma(\mathfrak{p})} \int_{0}^{1}(1-s)^{\mathfrak{p}-1} d s\right. \\
& \left.+\frac{1-t}{\Gamma(\mathfrak{p}-1)} \int_{0}^{1}(1-s)^{\mathfrak{p}-2} d s+\frac{1}{\Gamma(\mathfrak{p})} \int_{0}^{t}(t-s)^{\mathfrak{p}-1} d s\right)^{6} \\
& \leq \frac{1}{4^{6}} \sup _{\mathfrak{t} \in[0,1]}|y(\mathfrak{t})-w(\mathfrak{t})|^{6}\left[\frac{1-t}{\Gamma(\mathfrak{p}+1)}+\frac{1-t}{\Gamma(\mathfrak{p})}+\frac{t^{\mathfrak{p}}}{\Gamma(\mathfrak{p}+1)}\right]^{6} \\
& =\beta \sup _{\mathfrak{t} \in[0,1]}|y(\mathfrak{t})-w(\mathfrak{t})|^{6} \text {, }
\end{aligned}
$$


where $\beta=\sup _{\mathfrak{t} \in(0,1)} \frac{1}{4096}\left[\frac{1-\mathfrak{t}}{\Gamma(\mathfrak{p}+1)}+\frac{1-\mathfrak{t}}{\Gamma(\mathfrak{p})}+\frac{\mathfrak{t}^{\mathfrak{p}}}{\Gamma(\mathfrak{p}+1)}\right]^{6}$. Therefore, the above inequality becomes

$$
\begin{aligned}
\sup _{\mathfrak{t} \in[0,1]}|F y(\mathfrak{t})-F w(\mathfrak{t})|^{6} & \leq \beta \sup _{\mathfrak{t} \in[0,1]}|y(\mathfrak{t})-w(\mathfrak{t})|^{6} \\
\Rightarrow \alpha t+\frac{\gamma}{\beta} \sup _{\mathfrak{t} \in[0,1]}|F y(\mathfrak{t})-F w(\mathfrak{t})|^{6} & \leq \alpha t+\gamma \sup _{\mathfrak{t} \in[0,1]}|y(\mathfrak{t})-w(\mathfrak{t})|^{6} \\
\Rightarrow \frac{\alpha(\beta t)}{\alpha(\beta t)+\gamma \sup _{\mathfrak{t} \in[0,1]}|F y(\mathfrak{t})-F w(\mathfrak{t})|^{6}} & \geq \frac{\alpha t}{\alpha t+\gamma \sup _{\mathfrak{t} \in[0,1]}|y(\mathfrak{t})-w(\mathfrak{t})|^{6}} \\
\Rightarrow \mathfrak{m}_{\mathrm{h}}(F y, F w, \beta t) & \geq \mathfrak{m}_{\mathrm{h}}(y, w, t),
\end{aligned}
$$

for some $\alpha, \gamma>0$. Thereby, we observe that the assumptions of the Theorem 1 are fulfilled. Resultantly, $F$ has a unique fixed point; accordingly, the specified NFDE has a unique solution.

Example 3. Consider the fractional order differential equation

$$
D_{0+}^{2} y(t)=g(t, y(t)), 0<t<1,
$$

where $g$ is defined by

$$
g(t, y(t))=\frac{1}{4(1+y(t))}, \forall y \geq 0
$$

in addition to the boundary conditions

$$
y(0)+y^{\prime}(0)=0, y(1)+y^{\prime}(1)=0 .
$$

Proof. Let $F: X \rightarrow X$ be an integral operator defined as in Theorem 4 and $g(s, y(s))=$ $\frac{1}{4(1+y(s))}, y(s) \geq 0$ for $s \in[0,1]$.

(i) Notice that $g:[0,1] \times[0, \infty) \rightarrow[0, \infty)$ is continuous and

$$
\begin{aligned}
|g(s, y(s))-g(s, w(s))| & =\left|\frac{1}{4(1+y(s))}-\frac{1}{4(1+w(s))}\right| \\
& =\frac{|y(s)-w(s)|}{4(1+y(s))(1+w(s))} \leq \frac{|y(s)-w(s)|}{4} .
\end{aligned}
$$

(ii) In this example, we notice that $\mathfrak{p}=2$. Hence,

$$
\begin{aligned}
\beta=\sup _{\mathfrak{t} \in(0,1)} \frac{1}{4096}\left(\frac{1-\mathfrak{t}}{\Gamma(\mathfrak{p}+1)}+\frac{1-\mathfrak{t}}{\Gamma(\mathfrak{p})}+\frac{\mathfrak{t}^{\mathfrak{p}}}{\Gamma(\mathfrak{p}+1)}\right)^{6} & =\sup _{\mathfrak{t} \in(0,1)} \frac{1}{4096}\left(\frac{1-\mathfrak{t}}{\Gamma(3)}+\frac{1-\mathfrak{t}}{\Gamma(2)}+\frac{\mathfrak{t}^{2}}{\Gamma(3)}\right)^{6} \\
& =\sup _{\mathfrak{t} \in(0,1)} \frac{1}{4096}\left(\frac{\mathfrak{t}^{2}-3 \mathfrak{t}+3}{2}\right)^{6} \\
& =0.00278091<1 .
\end{aligned}
$$

Thereby, all the hypotheses of Theorem 4 are verified. Hence, the problem (40) has a solution on $X$.

\section{Conclusions}

In this manuscript, we have introduced the concept of fuzzy extended hexagonal $b$-metric spaces as a generalization of an extended fuzzy rectangular $b$-metric spaces [30], 
as well as given some associated examples, convergence of sequences, CS, and completeness of the FEHb-MS. Moreover, the Banach fixed point theorem, as well as several other fixed point theorems, was established in this space. As an application of our primary results, we investigate the existence and uniqueness of solutions to a nonlinear fractional differential equation (NFDE).

Author Contributions: Conceptualization, K.G.; Funding acquisition, T.A. and B.A.; Methodology, K.G., T.A. and B.A.; Supervision, T.A.; Validation, K.G.; Writing-original draft, S.T.Z. and K.G.; Writing-review \& editing, S.T.Z. and K.G. All authors read and agreed to the published version of the manuscript.

Funding: This research received no external funding.

Institutional Review Board Statement: Not applicable.

Informed Consent Statement: Not applicable.

Data Availability Statement: Not applicable.

Acknowledgments: The first and second authors would like to thank the Management of Sri Sivasubramaniya Nadar College of Engineering for their continuous support and encouragement to carry out this research work. The authors Bahaaeldin Abdalla and Thabet Abdeljawad would like to thank Prince Sultan University for paying the APC and for the support through the TAS research lab.

Conflicts of Interest: The authors declare no conflict of interest.

\section{References}

1. Zadeh, L.A. Fuzzy sets. Inf. Control 1965, 8, 338-353. [CrossRef]

2. Deng, Z. Fuzzy pseudometric spaces. J. Math. Anal. Appl. 1982, 86, 74-95. [CrossRef]

3. George, A.; Veeramani, P. On some results in fuzzy metric spaces. Fuzzy Sets Syst. 1994, 64, 395-399. [CrossRef]

4. Schweizer, B.; Sklar, A. Statistical metric spaces. Pac. J. Math. 1960, 10, 385-389. [CrossRef]

5. Schweizer, B.; Sklar, A. Probabilistic Metric Spaces; Courier Corporation: Amsterdam, The Netherlands, 1983.

6. Kramosil, I.; Michalek, J. Fuzzy metrics and statistical metric spaces. Kybernetika 1975, 11, 336-344.

7. Grabiec, M. Fixed points in fuzzy metric spaces. Fuzzy Sets Syst. 1988, 27, 385-389. [CrossRef]

8. Bakhtin, I.A.The contraction mapping principle in almost metric spaces. Funct. Anal. 1989, 30, $26-37$.

9. Boriceanu, M.; Petrusel, A.; Rus, I.A. Fixed points theorems for some multivalued generalized contraction in $b$-metric spaces. Int. J. Math. Stat. 2010, 6, 65-76.

10. Czerwik, S. Contraction mappings in b-metric spaces. Acta Math. Inform. Univ. Ostrav. 1993, 1, 5-11.

11. Kamran, T.; Samreen, M.; Ain, Q.U.L. A generalization of $b$-metric space and some fixed point theorems. Mathematics 2017, 5, 19. [CrossRef]

12. Mehmood, F.; Ali, R.; Ionescu, C.; Kamran, T. Extended fuzzy b-metric spaces. J. Math. Anal. 2017, 8, $124-131$.

13. Mehmood, F.; Ali, R.; Hussain, N. Contractions in fuzzy rectangular b-metric spaces with application. J. Intell. Fuzzy Syst. 2019, 37, 1275-1285. [CrossRef]

14. Saleem, N.; Hüseyin, I.; Salman, F.; Choonkil, P. Fuzzy double controlled metric spaces and related results. J. Intell. Fuzzy Syst. 2021, 40, 9977-9985. [CrossRef]

15. Sezen, M.S. Controlled fuzzy metric spaces and some related fixed point results. Numer. Methods Partial Differ. Equ. 2021, 37, 583-593. [CrossRef]

16. Salman, F.; Hüseyin, I.; Saleem, N. Fuzzy triple controlled metric spaces and related fixed point results. J. Funct. Spaces 2021, 2021, 9936992.

17. George, R.; Radenovic, S.; Reshma, K.P.; Shukla, S. Rectangular b-metric space and contraction principles. J. Nonlinear Sci. Appl. 2015, 8, 1005-1013. [CrossRef]

18. Mlaiki, N.; Aydi, H.; Souayah, N.; Abdeljawad, T. Controlled metric type spaces and the related contraction principle. Mathematics 2018, 6, 194. [CrossRef]

19. Abdeljawad, T.; Mlaiki, N.; Aydi, H.; Souayah, N. Double controlled metric type spaces and some fixed point results. Mathematics 2018, 6, 320. [CrossRef]

20. Sumaiya Tasneem, Z.; Kalpana, G.; Abdeljawad, T. A different approach to fixed point theorems on triple controlled metric type spaces with a numerical experiment. Dyn. Syst. Appl. 2021, 30, 111-130.

21. Kalpana, G.; Sumaiya Tasneem, Z. Some fixed point results in extended hexagonal $b$-metric spaces approach to the existence of a solution to fredholm integral equations. J. Math. Anal. 2020, 11, 1-17.

22. Abbas, M.; Lael, F.; Saleem, N. Fuzzy $b$-metric spaces : Fixed point results for $\Psi$-contraction Correspondences and Their Application. Axioms 2020, 9, 36. [CrossRef] 
23. Bashir, S.; Saleem, N.; Aydi, H.; Husnine, S.M.; Rwaily, A.A. Developments of some new results that weaken certain conditions of fractional type differential equations. Adv. Differ. Equ. 2021, 359, 2021.

24. Latif, A.; Saleem, N.; Abbas, M. $\alpha$-optimal best proximity point result involving proximal contraction mappings in fuzzy metric spaces. J. Nonlinear Sci. Appl. 2017, 10, 92-103. [CrossRef]

25. Saleem, N.; Abbas, M.; Raza, Z. Fixed fuzzy point results of generalized Suzuki type F-contraction mappings in ordered metric spaces. Georgian Math. J. 2020, 27, 307-320. [CrossRef]

26. Saleem, N.; Ali, B.; Abbas, M.; Raza, Z. Fixed points of Suzuki type generalized multivalued mappings in fuzzy metric spaces with applications. Fixed Point Theory Appl. 2015, 1, 1-18. [CrossRef]

27. Saleem, N.; Zhou, M.; Bashir, S.; Husnine, S.M. Some new generalizations of F-contraction type mappings that weaken certain conditions on Caputo fractional type differential equations. Aims Math. 2021, 6, 12718-12742. [CrossRef]

28. Asim, M.; Imdad, M.; Radenović, S. Fixed point results in extended rectangular $b$-metric spaces with an application. Upb Sci. Bull. Ser. Appl. Math. Phys. 2019, 81, 43-50.

29. Nădxaxban, S. Fuzzy b-metric spaces. Int. J. Comput. Commun. Control. 2016, 11, 273-281.

30. Badshah-e-Rome, M.; Sarwar, R.L. Rosana, Fixed point results via $\alpha$-admissibility in extended fuzzy rectangular $b$-metric spaces with applications to integral equations. Mathematics 2021, 9, 2009. [CrossRef]

31. Roshan, J.R.; Parvaneh, V.; Kadelburg, Z.; Hussain, N. New fixed point results in $b$-rectangular metric spaces. Nonlinear Anal. Model. Control. 2016, 21, 614-634. [CrossRef]

32. Mihet, D. Fuzzy $\Psi$-contractive mappings in non-Archimedean fuzzy metric spaces. Fuzzy Sets Syst. 2008, 159, 739-744. [CrossRef]

33. Zhang, S. Positive solutions for boundary-value problems of nonlinear fractional differential equations. Electron. J. Differ. Equ. 2006, 36, 1-12. [CrossRef] 